\title{
Temporal Variability in Southeast Asian Dragon Jars: A Case from the Philippines
}

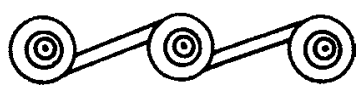

\author{
STEPHEN DUEPPEN
}

\section{INTRODUCTION}

VARIATIONS IN OBJECTS are the product of human actions, intentional or unintentional, through socially performed activities organized or carried out by diverse social actors and groups. The identification of the consistency (or inconsistency) of these actions can inform us about the nature of the social groups engaged in material culture production, as well as, indirectly, their consumers. By considering all actions taken on matter in the stages of production of an object, one can discern both broad technical practices as well as seemingly arbitrary choices made by the producers (Lemonnier 1992). The analysis presented in this article demonstrates the fruitful results obtained when these understandings are applied toward an archaeological data set, in this case stoneware jars found in second millennium A.D. mortuary contexts throughout the southern Philippines.

The large stoneware storage and transport jars produced in mainland Asia for the Southeast Asian trade networks of the second millennium A.D. comprise an infrequently studied class of objects (but see Grave and Maccheroni 2009). As containers, they facilitated the transport of ship provisions and trade items intended for the international market. In addition, archaeological, textual, and ethnographic evidence suggest that they played an important symbolic role in some Island Southeast Asian cultural contexts through their incorporation into local ritual and political systems. Despite their importance, little is known of the nature and social context of stoneware jar production, use, and consumption.

This article explores the diverse patterning found in a subset of glazed stoneware storage jars: dragon jars. These are large $(30-100 \mathrm{~cm}$ in height) brown-glazed stoneware vessels with applied handles on the shoulder. Their name derives from their adornment with incised, impressed, or plastic dragon, lion, demon, or floral motifs. Known to have been produced in mainland Asia, dragon jars were used in trade throughout insular Southeast Asia and the Indian Ocean. They have been recovered in archaeological sites throughout this vast region. The dragon jars in this analysis are derived from mortuary sites in the Philippines that were collected by Carl Guthe in

Stephen Dueppen is an Assistant Professor in the Department of Anthropology at the University of Oregon in Eugene, Oregon, U.S.A. 
the 1920s and are housed in the Guthe Collection at the University of Michigan Museum of Anthropology.

My analysis of these vessels was initially a typological exercise intended to systematically quantify variability in vessel form to identify coherent groups, which could be compared to results from chemical sourcing studies carried out by the Museum's Asian Archaeology division on a subset of the collection. I adopted a methodology focused on documenting the technical practices entailed in each stage of vessel production. ${ }^{1}$

This article presents the results of in-depth exploration of patterning in the defined groups. Synchronic and diachronic variability within and between groups of jars was analyzed. Temporal trends and production locales advanced for dragon jar production were tested against the Guthe Collection to explore patterns across space and site type. The result is a usable chronology based upon a completely described set of types that will allow additional studies of mortuary practice in the Philippines, and be valuable to scholars studying these important and poorly understood ceramics in other regions of the world.

\section{SOCIAL PRODUCTION OF TECHNOLOGY}

My work begins from the fundamental premise that technologies, defined as "all aspects of the process of action on matter" (Lemonnier 1992:1), are social productions (Lemonnier 1992; Leroi-Gourhan 1943, 1945; Mauss 1954). Material culture studies can address many questions pertaining to the larger social context in which objects are embedded through examination of the physical indicators of the locus, organization, and characteristics of production, use, and final deposition.

Past research on the social nature of technologies has suggested that social representations, or shared sets of understandings among members of a given social group, influence the development and performance of technological actions (Lemonnier 1992; Leroi-Gourhan 1943, 1945). Since members of social groups (of variable scale and composition) tend to do things in a certain way at specific times, different "traditions of doing" can be discerned through the identification of points or dimensions of variation in the production process. The degree of standardization in this process may vary with the particular social or political characteristics of the group and with the particular dimension of variation under consideration.

Variability in the production of material culture is therefore the result of socially mediated choices in individual human action. These choices range from the gestures used in action on matter (when and how they are used and by whom) to the raw materials collected and prepared to the types of tools employed (Leroi-Gourhan 1943; see also Lemonnier 1992). Following Leroi-Gourhan (1943), variability in all of these is limited by both the action-on-matter function that an object is intended to serve and the above-mentioned temporally and spatially specific social constraints. In short, choices made concerning the material potentials and characteristics of the end product are linked to both cultural characteristics and the local or regional environment in which production occurs. The influence of the social environment cannot be underestimated: an object's social function may influence how producers shape it, even to the point that its final form seems detrimental to the efficiency of its action-on-matter function (Lemonnier 1992). In addition, the use and discard of objects can also result in action-on-matter effects (e.g., reshaping, decay, burning). The result is that all aspects of production, use, and discard are socially relevant and many are technologi- 
cally visible. This general point about the social relevance of all variability in material culture is mirrored in the long-standing archaeological debate on style, which accepts that formal variation in material culture can transmit information both consciously or unconsciously (Hegmon 1992; Stark 1998; Wiessner 1983, 1984, 1985; Wobst 1977). The anthropological questions then become: How does a technology operate socially in a specific cultural context? What is the social context for identified variability?

\section{RECOGNIZING VARIABILITY IN MATERIAL PRODUCTION}

Building from above, a useful way to characterize the range of variation in a single class of finished objects is to delineate operational chains of production involved in their generation, that is, the "series of operations involved in any transformation of matter (including our own body) by human beings" (Lemonnier 1992:26). Such an analysis identifies which components of the process created the final and often subtle differences between individual objects or classes of objects. Since technological actions are often repetitive, the analysis of large samples of objects permits the delineation of the organizing principles behind what may visually appear to be diverse choices.

Discerning operational chains in an archaeologically derived collection is difficult, since data may be incomplete or compromised due to site formation processes or recording methodologies. Nevertheless, in many cases, useful and comparable information can be found for many individual production stages. One advantage of this method for defining classes of objects is that every recorded variable in the study is treated as the consequence of a socially performed act in a particular stage of production. Thus, even the smallest variable is kept in a social context. Grouping socially relevant variables allows researchers to delineate socially relevant "types." These understandings can then be brought to bear on the search for patterns in the use and distribution of objects.

\section{DRAGON JARS AND THE PHILIPPINES}

Large stoneware storage jars served to transport commercial goods (often fragile items), or provisions on long sea voyages throughout Southeast Asia during the second millennium A.D. (Desroches et al. 1996:228-229; Valdes 1993; Valdes and Alba 1993:38-42; Valdes et al. 1992:26). For example, evidence from the Spanish shipwreck the San Diego (A.D. 1600) revealed various uses of stoneware jars, including pickled-meat storage, containers for the transport of fine ceramics and, possibly, water storage (Desroches et al. 1996:228-229). Dragon jars were just one class of transport jars used in the region. In their second life in the Philippines they were also important for local social and political relations. What were the regional trends that influenced the transport of dragon jars to the Philippines?

Interregional trade in Southeast Asia during the second millennium A.D. was a complex process involving the material desires of a wide variety of participants and cultures of varying social and political complexity, from Chinese and Spanish empires to dynamic state polities in Thailand, the Vietnamese coast, and Indonesia, to the chiefdoms and non-hierarchical societies of the Philippines and other regions of Island Southeast Asia. The Philippine archipelago had been involved in low-level regional interactions for thousands of years (Solheim 2002). However, expanded trade 
networks evolved throughout the second millennium A.D., tying various chiefdoms to larger interregional commerce (Diem 2004; Junker 1999). Archaeological and historical records show evidence of significant indirect Chinese trade (via Malay and Arab traders) with the Philippines starting around A.D. 1000, concurrent with the Song dynasty in China (Fox 1967; Junker 1999). Tributary polities (e.g., Champa and Borneo) along trade routes exchanged Chinese products such as porcelains, fine metals, and other luxury goods for raw materials derived from the highlands and islands, including spices, forest products, and metals (Diem 2004; Junker 1999). Junker (1999) has suggested that occasional commerce likely occurred directly between China and the Philippines, but Filipino attempts to establish direct trade relations with China during this period were denied as Chinese efforts generally focused farther west (Diem 2004: 475). Early Song period trade can thus be characterized as indirect and relatively low volume, but involving highly diverse luxury goods (Junker 1999).

Direct trade between China and the Philippines began in the late twelfth century (concurrent with the late Song Dynasty) with the shift from an indirect trade route along the Vietnamese coast to a more direct route from Fujian ports to Taiwan and the Philippines (Diem 2004; Junker 1999; Valdes 1992). By the thirteenth century (late Song and Yuan dynasties), Chinese junks joined Malay ships in these networks at the expense of the polities formerly favored in the indirect tributary system (Junker 1999). The Chinese again exchanged finished products (e.g., porcelain, trade gold, iron pots) for raw materials (e.g., beeswax, cotton, pearls, tortoise shell) (see Junker 1999:196 for complete lists).

Commerce conducted by licensed Chinese merchants was reorganized in A.D. 1371, shortly after the installation of the Ming dynasty in A.D. 1368 (Reid 1993). The centralization of trade entailed a general (though difficult to enforce) ban on private trade and was mirrored in production that was more closely directed by the Chinese Empire, resulting in the manufacture of lower quality, more homogenous products for the international market (e.g., porcelains became coarser and more standardized) (Fox 1967). In order to regulate trade, the Chinese formalized relations with specific trading partners in the Philippines by establishing tributary relations with Philippine chiefs (Junker 1990, 1999; Reid 1993; Valdes et al. 1992) as they had earlier with polities along the Vietnamese coast (Diem 2004). The result was a period of particularly intense interactions between the Philippines and China between A.D. 1372 and 1427 (Diem 2004). In short, the new centralization of control over and general expansion of commerce resulted in mass production of trade goods delivered to specific trading partners.

By the early fifteenth century, the number of licensed Chinese merchants could not keep up with the growing demands of Philippine and other Island Southeast Asian consumers. This was due to a generally anti-foreign political environment in China. The "Ming Gap" (Brown 2004) prompted the expansion of trade between Mainland Southeast Asia (Viet Nam, Thailand) and the Philippines and the development of commercial zones in coastal Mainland Southeast Asia, resulting in "complex interconnected regional networks" (Junker 1999:195). Champa, a loose affiliation of state-level polities located along the central Vietnamese coast (Vickery 2009), were important participants in this network (Diem 2004). By 1567, when China officially lifted the ban on private trade, large numbers of non-Chinese products had already become long embedded in the Philippine market (Reid 1993). The diversification of luxury good producers is not surprising given the many Southeast Asian complex 
societies that arose during the second millennium A.D., as well as the arrival of European influence in the region during the sixteenth century.

\section{ARCHAEOLOGICAL EVIDENCE FOR TRADE IN THE PHILIPPINES}

Archaeological research suggests that prior to A.D. 1000, foreign materials were relatively unimportant in the social and political economy of Philippine chiefdoms. Locally produced prestige goods, including earthenware, metal, and glass beads, were used in rituals (e.g., receptions, marriages, rites of passage, mortuary rites) and played an important part in building alliances (Bacus 1995; Barbosa 1992; Junker 1999: 168). After A.D. 1000, foreign-made products began to replace locally produced socially and politically charged objects. Mortuary data from the cemetery of Santa Ana on the island of Luzon show that Chinese celadons and other glazed stonewares and porcelains supplanted local objects in highly ranked burials of the eleventh through fourteenth centuries A.D. (Junker 1999:171-175).

By the fifteenth to sixteenth centuries, concurrent with the Chinese Ming dynasty, foreign-made goods were pervasive and diverse in elite mortuary contexts. They also extended into lesser elite and commoner burials. There may have been a ten-fold increase in foreign ceramics reaching the Philippines (Junker 1990, 1999:198). According to Fox (1959:355), at the fifteenth-century Calatagan mortuary sites of Kay Tomas and Pulong Bakaw in Batangas Province, southwest Luzon, 50 percent of burials contained foreign-produced goods; Thai and Vietnamese ceramics were widely distributed, while Chinese products were relatively restricted (see also Junker 1999).

The increasing diversity of trade relations is reflected in data that show that some fifteenth-century southern Philippine settlement sites contained 20 to 40 percent Thai and Vietnamese stonewares and porcelains in their foreign assemblages (Junker 1999:202). These data suggest that people in the Philippines were increasingly participating in interregional trade networks with an increasing diversity of trade partners.

\section{THE ROLE OF DRAGON JARS}

Historically in the Philippine context, large stoneware jars, especially decorated dragon jars, were more than containers for holding commerical goods. They were foreign-produced and symbolically charged items that were used to store rice wine and beer used in rituals such as births, deaths, and weddings (Barbosa 1992; Desroches et al. 1996). Even today, some groups treat these rare and prestigious heirloom vessels with respect (Barbosa 1992; Harrisson 1986). Scholars have suggested that the integration of foreign-produced dragon jars into Filipino societies was aided by two factors. First, since locally produced jars were traditionally used in rituals, the added prestige of foreignness and increased durability made imported stoneware jars even more symbolically powerful (Barbosa 1992:70-74). Second, the symbolic and potential political power of exotics was expressed in decorative elements such as dragons, floral designs, and other images that were not found in Philippine ceramic traditions. However, it has been suggested that the importance of dragons may have been derived from their association with traditional Philippine symbols (e.g., serpents) (Long 1992: 25). 
Archaeologically, dragon jars are found as grave goods in mortuary contexts dating between the twelfth and nineteenth centuries in the Philippines. The importance of maintaining access to sources of jars may have been because of the political advantage of controlling the acquisition and distribution of essential ritual objects. Even though they are not the fine porcelains valued throughout Asia, dragon jars may be seen as a form of capital in the political economies of some Philippine chiefdoms. Some recent Filipino societies even had complex ranking systems for jars, reflecting political and social differentiation (Desroches et al. 1996:228-229).

Evidence from the A.D. 1600 San Diego shipwreck off Luzon provides insight into the significance of decorated stoneware jars within past Philippine mortuary rituals (Valdes and Alba 1993:43). Of the 621 storage jars on board the San Diego, only 5.3 percent were dragon jars. In contrast, more than 50 percent of the large glazed stoneware jars in the Guthe Collection are dragon jars. This suggests that decorated jars may have been incorporated into mortuary contexts at far higher frequencies than undecorated stoneware jars, perhaps pointing to their higher cultural value to indigenous Philippine consumers.

While a considerable amount is known about the historic consumption contexts of these jars, relatively little research has been devoted to understanding the dynamic role of dragon jars in Southeast Asian societies. Since jars were necessary to transport goods from multiple production centers, it is likely that each complex Mainland Southeast Asian commercial center or polity either produced their own or secured access to storage jars made at one or more large-scale production centers. A study of dragon jar production may therefore contribute significantly to our understanding of international commercial competition between Chinese ports and other Southeast Asian centers during this period, as well as more local developments in the islands.

\section{THE GUTHE COLLECTION}

The Philippine Expedition or "Guthe" Collection at the University of Michigan Museum of Anthropology is derived from fieldwork carried out by Carl Guthe, the museum's founder and first curator of the "Division of the Orient." Between 1922 and 1925, Guthe recovered archaeological remains from 542 mortuary sites in the southern half of the Philippine archipelago (Sinopoli, this volume). He documented and collected over 13,000 objects, including earthenwares, stonewares, porcelains, beads, and metals. Relevant for this study, the collection contains over 200 large stoneware jars, of which roughly half $(\sim 102)$ are dragon jars. Most $(\sim 85)$ are fragmentary, but 17 are complete. This study focuses on 63 vessels chosen for the completeness of their diagnostic traits. All vessels are provenienced to site, but as in many collections from the early twentieth century, the sample lacks detailed provenience and contextual information. The current documentation system includes the original Guthe Collection numbers (e.g., G-128), an UMMA catalog number (e.g., \#34472), and also a CMS number (e.g., CMS 001) from Instrumental Neutron Activation analyses performed on a subset of this collection. These numbers will be referenced throughout the paper.

The University of Michigan's Asian Archaeology Division has been involved since 1995 in studying the dragon jar collection as part of a general program initiated by curator Carla Sinopoli to fully characterize the Guthe Collection. Researchers Stephen Dueppen, Robert Brubaker, Christophe Descantes, Michael D. Glascock, Will Griffin, Hector Neff, Rasmi Shoocongdej, and Robert Speakman have studied the 
composition, style, and technology of the dragon jar collection (Sinopoli et al. 2006). My primary research focus, as discussed in this article, has been on stylistic and technological analyses. The results of the compositional analyses are integrated throughout the article.

The study of a fragmentary collection of this sort requires flexibility. While problems of provenience and sampling do not necessarily cripple a study of production, the limitations of various features of the sample influenced the possible types of data recorded. For example, data on vessel form was limited due to a general lack of reconstructable body and base potsherds. Consequently, not all variables could be recorded for many of the potsherds. The following analysis is thus based on those characteristics that were the most common and therefore comparable.

\section{THE LIFE CYCLE OF A DRAGON JAR}

The life cycle of a dragon jar can be conceptualized as following a general sequence of seven basic stages (Table 1). Each stage is described below with a short description of relevant analyses. Each of the stages can be further divided into substages.

\section{Stage 1: Procurement of Raw Materials (Clay, Temper, and Materials for Decoration)}

Raw materials constitute the basic building blocks for creating a vessel. Much variability is expected in their procurement, as multiple functionally equivalent choices are often available in the environment. Variation may be found in color, texture, and density of the raw clay; chemical/mineralogical composition of the clay; temper material and size; and chemical composition of glaze due to variability in the recipe's raw ingredients.

Three analyses were performed on the dragon jar collection to discern variability in Stage 1. To characterize the paste composition (including clay and nonplastic inclusions), samples were submitted to Instrumental Neutron Activation Analysis (INAA). To characterize the glaze composition, samples were subsequently submitted to laserablation inductively coupled-plasma mass spectrometry analysis (LA-ICP-MS). To outline variability in the inclusions, either thin sections or clean breaks from potsherds from all four types described below were analyzed. In addition, petrographic

Table i. General Operational Chain for Dragon Jar Production

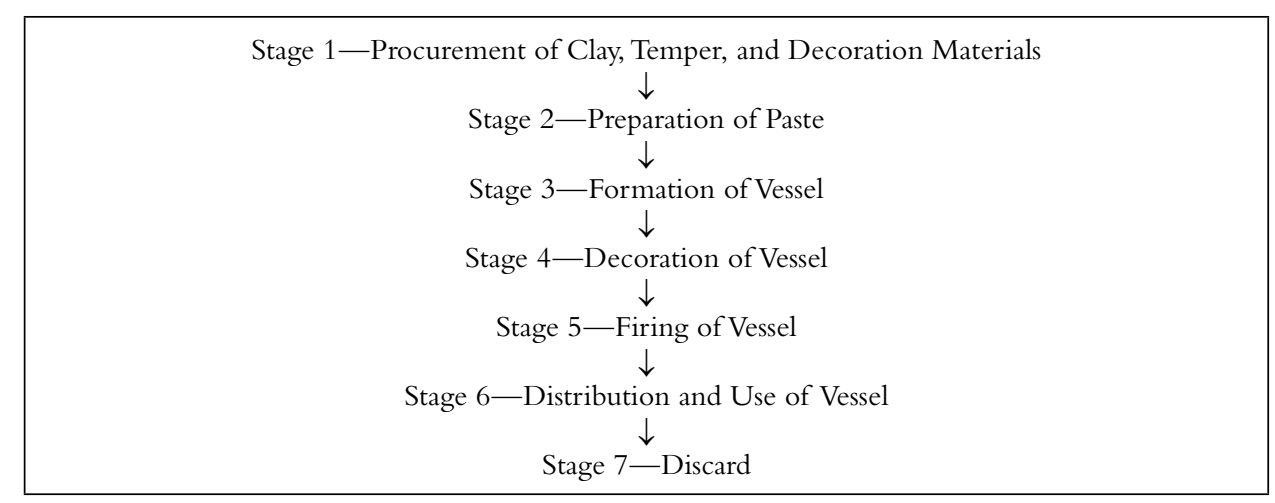


thin sections were prepared of the samples subject to chemical characterization, but their analysis is not included here.

\section{Stage 2: Paste Preparation}

This stage combines raw materials into a mixture that permits vessel shaping appropriate to the forming technology used. Variation may be found in the types and relative frequency of all the raw materials noted in Stage 1 above and the density/porosity of paste and inclusions.

To characterize the treatment of the paste made from the raw materials, a thin section/clean break was made in order to examine the density and inclusion distributions from the collection. Since Stage 1 and Stage 2 are analytically related, they are discussed together below.

\section{Stage 3: Vessel Formation}

There is potential for great variability in techniques in this stage, as potters seek to create vessels with specific forms and properties, often appropriate to an anticipated set of functions. Variation may be found in formal characteristics such as shape, thickness, angles, diameters, and so on and in vessel formation techniques such as coils, slabs, wheel, and mold.

To characterize vessel formation practices, rim forms were recorded when available and vessel thickness was systematically recorded for each vessel; however, data on vessel body formation were rare. Handles were all composed of clay strips that were attached to the vessel in the same manner, despite differences in orientation (vertical/ horizontal) or decoration (see next stage). Handles were primarily used to attach or tie lids to the vessel top.

\section{Stage 4: Vessel Decoration}

Decoration is here defined as all external treatment of a vessel, including some features such as handles that are also functional for jar usage. Decorations on dragon jars were divided into three classes: primary motifs, lug handles attached to the shoulder/neck, and glaze. Variation may be found in the techniques employed in the plastic decoration of each vessel, including sequence, location, and form of decorative treatments and in glaze application.

An extensive study of decorative techniques was performed recording the sequence (order) of techniques responsible for all decorations.

\section{Stage 5: Firing of Vessels}

Variation in firing techniques may be observed through both direct and indirect means. The pottery itself provides indirect evidence of technique since different temperatures and atmospheres of firing have different effects (stoneware, earthenware, porcelain, color and texture of glaze or surface). In this case, stoneware was chosen for functional reasons (i.e., its impermeability). More directly, kilns and associated wasters provide information on firing technology, scale, and types of ceramics produced. Recent evidence from the Vietnamese-Japanese excavations in Central Viet Nam (e.g., 
of the Go Sanh kilns) is assessed below (Aoyagi 2002; Diem 1999, 2004; Koezuka et al. 1996; Morimoto and Ohashi 2002; Yamamoto et al. 1993).

\section{Stage 6: Vessel Distribution/Use}

Direct evidence for dragon jar usage is found in shipwrecks and potentially in domestic contexts. Mortuary use of the vessels is considered a category of discard, so is discussed below. This article assesses available shipwreck data and historical evidence relevant to this stage, which is considered the primary use-life of these vessels. Currently, evidence (i.e., through residue analysis or spatial location in archaeological sites) for the use of dragon jars in the Philippines prior to discard is not available.

\section{Stage 7: Discard}

Dragon jars are found in large parts of Asia and the Indian Ocean in archaeological contexts; others remain in use in household contexts. A portion of dragon jars has been deposited in mortuary sites in the Philippines (including those in the Guthe Collection). Thus, in this study, my analysis of this stage is largely limited to the Guthe Collection disposal, which can be characterized as ritually based discard.

\section{DRAGON JAR TYPES}

In this section, I present my typological analysis of the Guthe Collection dragon jars. Four dragon jar groups were identified; they are presented holistically based upon evidence for production Stages 1-4. Evidence for each stage yielded highly redundant patterning; each jar type can be most easily defined by a unique operational chain for primary motif production, that is, the dragon or floral designs that appear on the neck, shoulder, or body of each vessel (Table 2). ${ }^{2}$ The results of my analysis of handle decoration were similar to those for primary motifs (Table 3 ).

Table 2. Primary Motif Production Sequences

\begin{tabular}{|c|c|c|c|}
\hline $\begin{array}{l}\text { SEQUENCE \# I } \\
\text { MOLD-IMPRESSED } \\
\text { TECHNIQUE }\end{array}$ & $\begin{array}{c}\text { SEQUENCE \#2 } \\
\text { MOLD-ATTACHED, IMPRESSED, } \\
\text { AND INCISED TECHNIQUE }\end{array}$ & $\begin{array}{c}\text { SEQUENCE \#3 } \\
\text { COIL AND INCISED } \\
\text { TECHNIQUE }\end{array}$ & $\begin{array}{c}\text { SEQUENCE \#4 } \\
\text { MOLD-ATTACHED AND/OR } \\
\text { IMPRESSED TECHNIQUE }\end{array}$ \\
\hline $\begin{array}{c}\text { Mold produced } \\
\text { (Ornate) } \\
\downarrow \\
\text { Mold impressed } \\
\text { into vessel } \\
\text { shoulder } \\
\downarrow \\
\text { Glaze over } \\
\text { motif }\end{array}$ & $\begin{array}{c}\text { Mold produced } \\
\text { (Simple body outline) } \\
\downarrow \\
\text { Clay impressed into mold, } \\
\text { then attached to vessel } \\
\text { shoulder or body } \\
\downarrow \\
\text { 1. Incised decorations on } \\
\text { and around dragon. } \\
\text { 2. Impressed semicircles for } \\
\text { scales } \\
\downarrow \\
\text { Glaze over motif }\end{array}$ & $\begin{array}{c}\text { Thin clay coils } \\
\text { hand-produced } \\
\downarrow \\
\text { Separate coils attached } \\
\text { and combined to } \\
\text { create plastic dragon } \\
\downarrow \\
\text { Parallel } 45 \text { degree } \\
\text { incisions made on } \\
\text { dragon body } \\
\downarrow \\
\text { Glaze over motif }\end{array}$ & $\begin{array}{c}\text { Mold produced } \\
\text { (Multiple types—ornate) } \\
\downarrow \\
\text { Clay impressed into } \\
\text { mold, then attached to } \\
\text { vessel shoulder or main } \\
\text { body } \\
\text { or } \\
\text { Mold impressed into } \\
\text { vessel body } \\
\downarrow \\
\text { Glaze over motif }\end{array}$ \\
\hline
\end{tabular}


Table 3. Handle Production Sequences

\begin{tabular}{|c|c|c|c|}
\hline $\begin{array}{l}\text { SEQUENCE \# I } \\
\text { MOLD-IMPRESSED AND } \\
\text { INCISION TECHNIQUE }\end{array}$ & $\begin{array}{c}\text { SEQUENCE \#2 } \\
\text { MOLD APPLICATION, INCISION, } \\
\text { AND IMPRESSION TECHNIQUE }\end{array}$ & $\begin{array}{c}\text { SEQUENCE \# } 3 \\
\text { VERTICAL INCISION } \\
\text { TECHNIQUE }\end{array}$ & $\begin{array}{c}\text { SEQUENCE }_{4} 4 \\
\text { HORIZONTAL } \\
\text { INCISION TECHNIQUE }\end{array}$ \\
\hline $\begin{array}{c}\text { Mold produced } \\
\downarrow \\
\text { Clay strip produced } \\
\downarrow \\
\text { Handle decorated: } \\
\text { mold impressed into } \\
\text { strip, vertical lines } \\
\text { incised above and } \\
\text { below impression } \\
\downarrow \\
\text { Handle attached to } \\
\text { vessel (vertical) } \\
\downarrow \\
\text { Glaze over handle }\end{array}$ & $\begin{array}{c}\text { Mold produced } \\
\downarrow \\
\text { Clay strip produced } \\
\downarrow \\
\text { Vertical channels made in } \\
\text { clay strip } \\
\downarrow \\
\text { Handle attached to vessel } \\
\text { (vertical) } \\
\downarrow \\
\text { Additional clay impressed } \\
\text { into mold and applied by } \\
\text { hand to handle strip } \\
\downarrow \\
\text { Handle decorated with } \\
\text { incisions/impressions } \\
\text { (optional)* } \\
\downarrow \\
\text { Glaze over handle }\end{array}$ & $\begin{array}{c}\text { Clay strip produced } \\
\downarrow \\
\text { Vertical incisions } \\
\text { cut into strip } \\
\downarrow \\
\text { Handle attached to } \\
\text { vessel (vertical) } \\
\downarrow \\
\text { Glaze over handle }\end{array}$ & $\begin{array}{c}\text { Clay strip produced } \\
\downarrow \\
\downarrow \\
\text { Horizontal incisions } \\
\text { cut into strip } \\
\downarrow \\
\text { Handle attached to } \\
\text { vessel (horizontal) } \\
\downarrow \\
\text { Glaze over handle }\end{array}$ \\
\hline
\end{tabular}

*The special dragon handles lack vertical incisions, but various features are incised and semicircular scales are impressed, as in the primary motif.

The evidence from various other stages of the production sequence is also informative, revealing patterns that are less obvious than decoration, but that were relevant to discerning variability in the collection. These patterns aided in the analysis of provenience and synchronic and diachronic variability in production, use, and discard, which follows the presentation of the four "production traditions" typologically identified for dragon jars (Table 4).

\section{Summary of Tradition 1 Jars}

The primary motifs of Tradition 1 jars are illustrated in Figure 1 and their production is summarized in Table 5 . These jars $(\mathrm{N}=11)$ are composed of a dense gray paste with few inclusions, have elongated and everted rims that are rounded (beaded) on the end, with rim diameters between 14-16/16-21 cm (interior/exterior), and are only $5.6 \mathrm{~mm}$ thick. Tradition 1 jars are shaped as inverted long convex cones in two size classes with heights of c. 33 and $67 \mathrm{~cm}$. Thin, snake-like dragons with horns on the head and a crest behind the face, whiskers beneath the chin, and four legs with three claws each are impressed on vessel shoulders with molds or stamps. Likewise, six vertical handles with mold-impressed dragon or devil faces surrounded by incisions are attached between the neck and shoulders of the each vessel. They are covered with an olive-brown glaze.

While Tradition 1, even more so than the other traditions discussed below, is very tightly defined within a narrow range of variation, a dragon jar from a domestic context at the fifteenth-century site of Kingany I-1 in northwestern Madagascar provides 
an interesting comparative case (Vérin 1972:293-301, fig. 90). Despite numerous formal and decorative similarities to Tradition 1 jars from the Guthe Collection, the Kingany vessel has significant differences including a right-facing dragon, only three handles, a different handle motif, and a smaller size. The characteristics of the Kingany vessel are presented in a separate column in Table 4 and for comparative purposes in Table 5. As discussed below, this alternative Tradition 1 jar was produced in a different location from the main group, and consequently provides evidence of the complexity of dragon jar production.

\section{Summary of Tradition 2 Jars}

The primary motifs of Tradition 2 jars are illustrated in Figure 2 and their production is summarized in Table 6 . These jars $(\mathrm{N}=35)$ are composed of gray pastes with a variety of densities and inclusions, have triangular-shaped everted rims with diameters between 11-20/12.5-25 cm (interior/exterior), and an average thickness of $8.2 \mathrm{~mm}$. They have mold-attached (sprigged, formed as a separate element in a mold and then attached to the vessel body or shoulder) dragons and horse-dragons with incised and impressed embellishments. Whiskers, facial features, and fins on the back are made by incision; scales are made by semicircular impressions; there are four feet with three toes each. Ten vessels have incised floral motifs next to the dragons. These decorations are always oriented left.

Three main classes of vertical handle are associated with this group. The first is a dragon-body handle that is basically an extension of the primary motif and is thus embellished with incisions and semicircular impressions (see dragon handle \#34473 in Figure 2). The second and most common type of handle was produced following a different sequence. The composite technique combined a strap handle with a moldformed plastic motif (see dog/dragon handle \#19924 in Figure 2). An example of a third class of handle, the fish handle (\#18194), is shown in Figure 2. It combines the operational sequence for the primary motif with that from the second handle class, combining a mold-formed face to the base of a thick coil of clay, which is then embellished with channels, incisions, and semicircular impressions similar to the primary motif. In addition to the fish handle, a common handle in this class is the lion handle, which has an attached face and gouges along the handle rather than incisions and impressions (Quimpo 1982:47). Tradition 2 vessels are covered most frequently with an olive-brown glaze.

\section{Summary of Tradition 3 Jars}

The primary motifs of Tradition 3 jars are illustrated in Figure 3 and their production is summarized in Table 7 . These jars $(\mathrm{N}=6)$ are composed of gray/pink pastes with a variety of densities and inclusions. Their rims (diameter 17-20.5 cm interior/23.5$29 \mathrm{~cm}$ exterior) are flared eversions with a beaded lip turned downward or straightnecked with slightly everted beaded lips, and vessels only $6 \mathrm{~mm}$ thick. The dragons applied to the shoulder of the vessel are built from hand-formed coils and decorated with diagonal incisions or gouges on the head and body. The dragons are made with an amalgam of coils, a big coil for the body and smaller ones for the appendages and head. They have horns, but no whiskers, and four legs with claws that have a "rootlike" appearance. The vessels have six vertical handles with simple vertical incisions. 


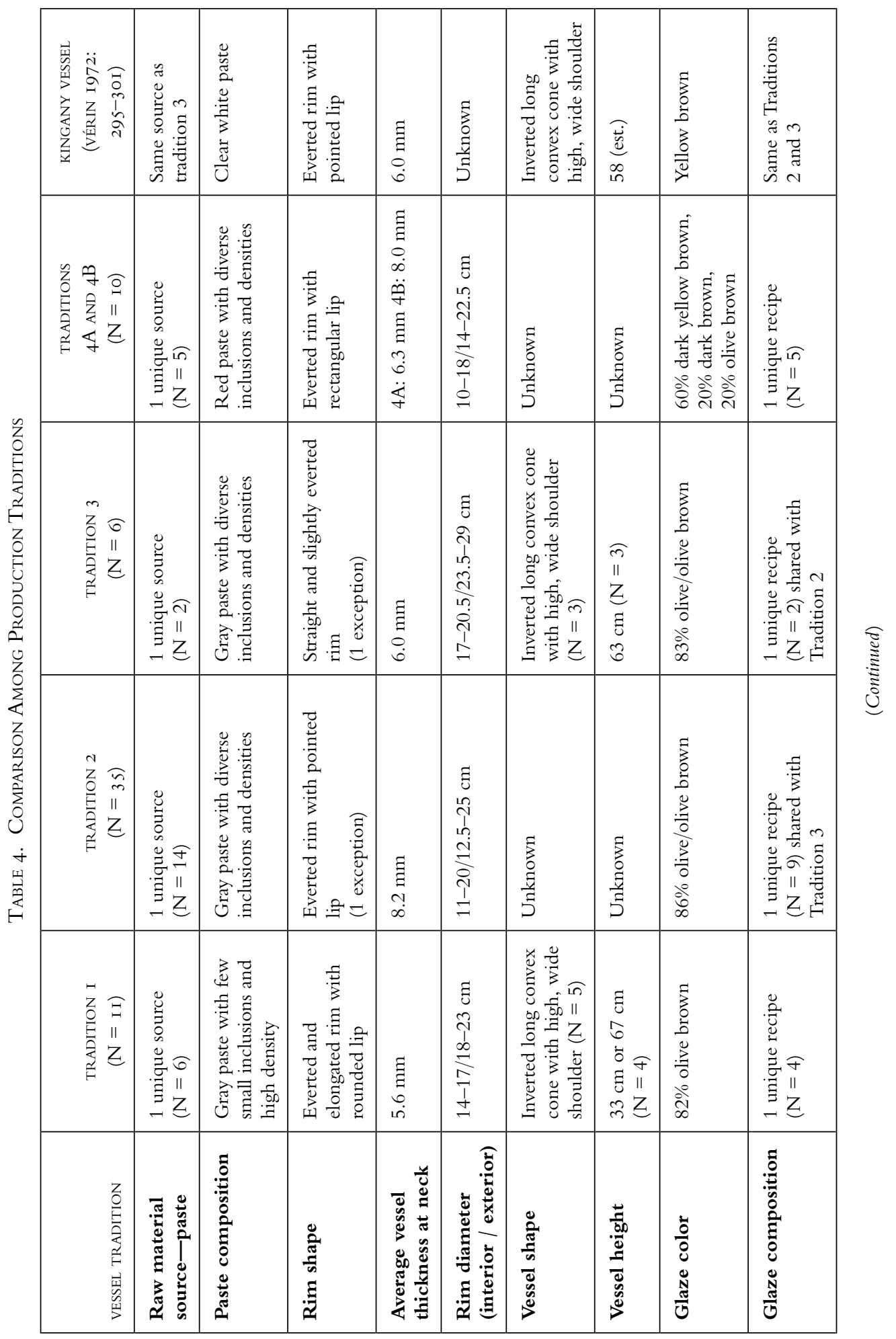




\begin{tabular}{|c|c|c|c|c|c|c|c|c|}
\hline 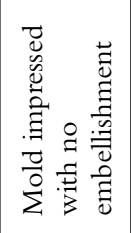 & 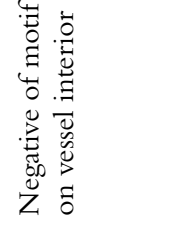 & 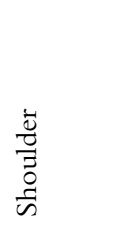 & 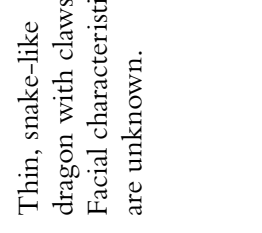 & 䓂 & 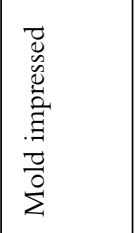 & 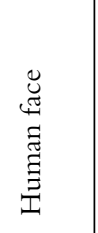 & $\overrightarrow{\widetilde{J}}$ & $m$ \\
\hline 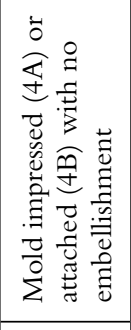 & 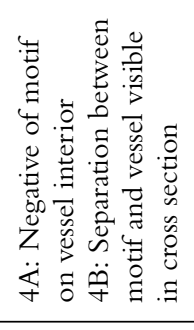 & 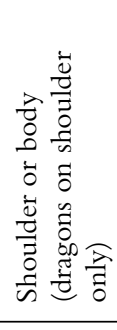 & 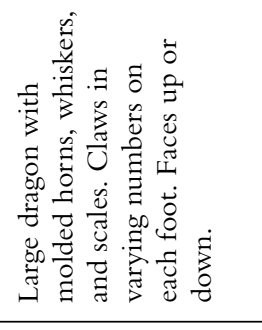 & 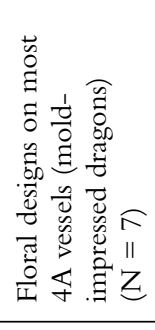 & 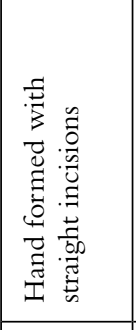 & 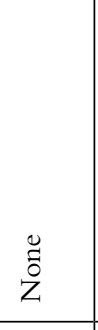 & 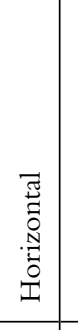 & $\begin{array}{l}\text { 言 } \\
\frac{\partial}{3} \\
\frac{3}{5}\end{array}$ \\
\hline 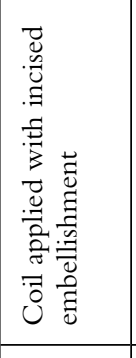 & 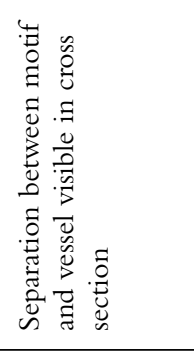 & 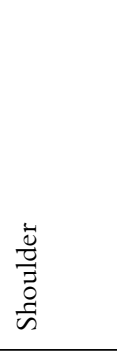 & 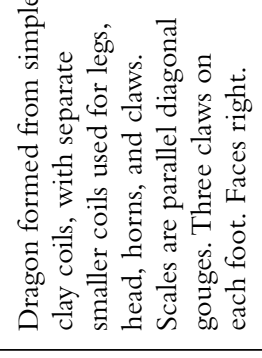 & 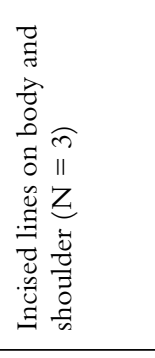 & 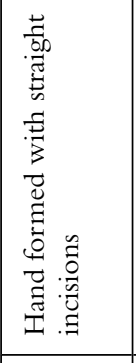 & 号 & $\begin{array}{l}\overrightarrow{5} \\
\overrightarrow{5} \\
\end{array}$ & $\begin{array}{l}\widehat{\pi} \\
\text { II } \\
z \\
0\end{array}$ \\
\hline 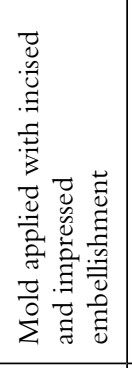 & 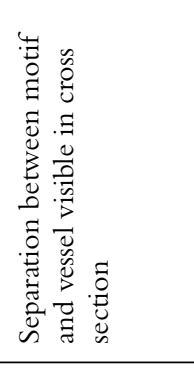 & 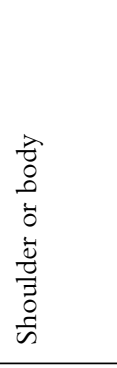 & 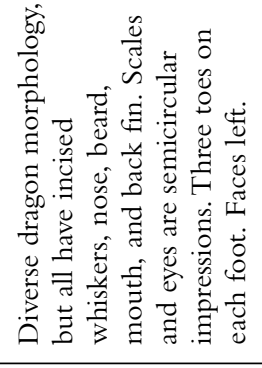 & 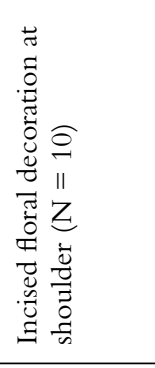 & 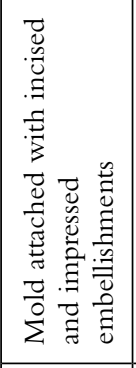 & 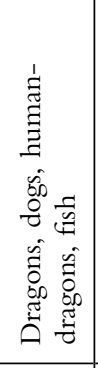 & 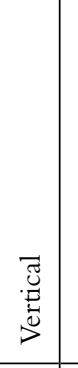 & 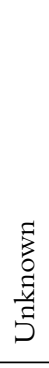 \\
\hline 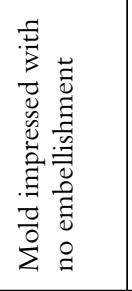 & 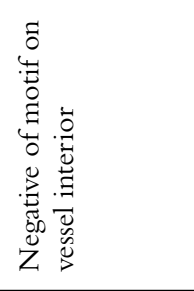 & 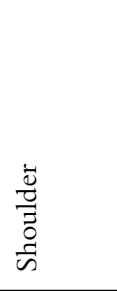 & 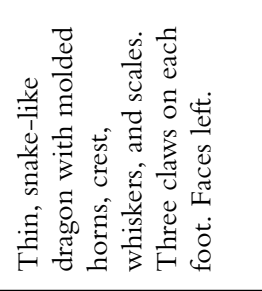 & 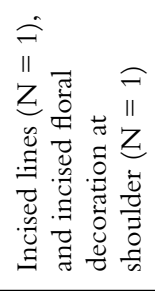 & 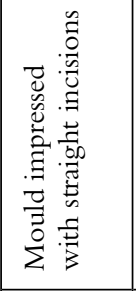 & 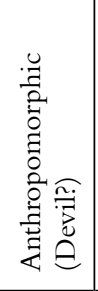 & 窇 & $\begin{array}{l}\text { f } \\
\text { II } \\
\text { z } \\
\end{array}$ \\
\hline 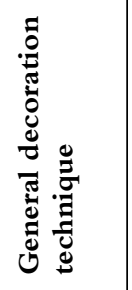 & 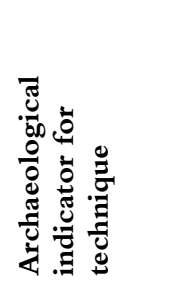 & 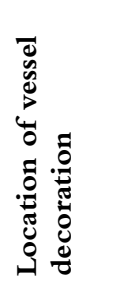 & 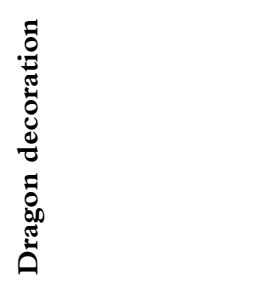 & 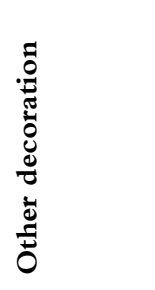 & 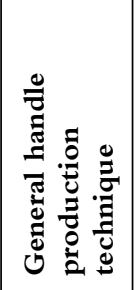 & 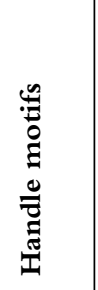 & 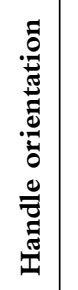 & \\
\hline
\end{tabular}



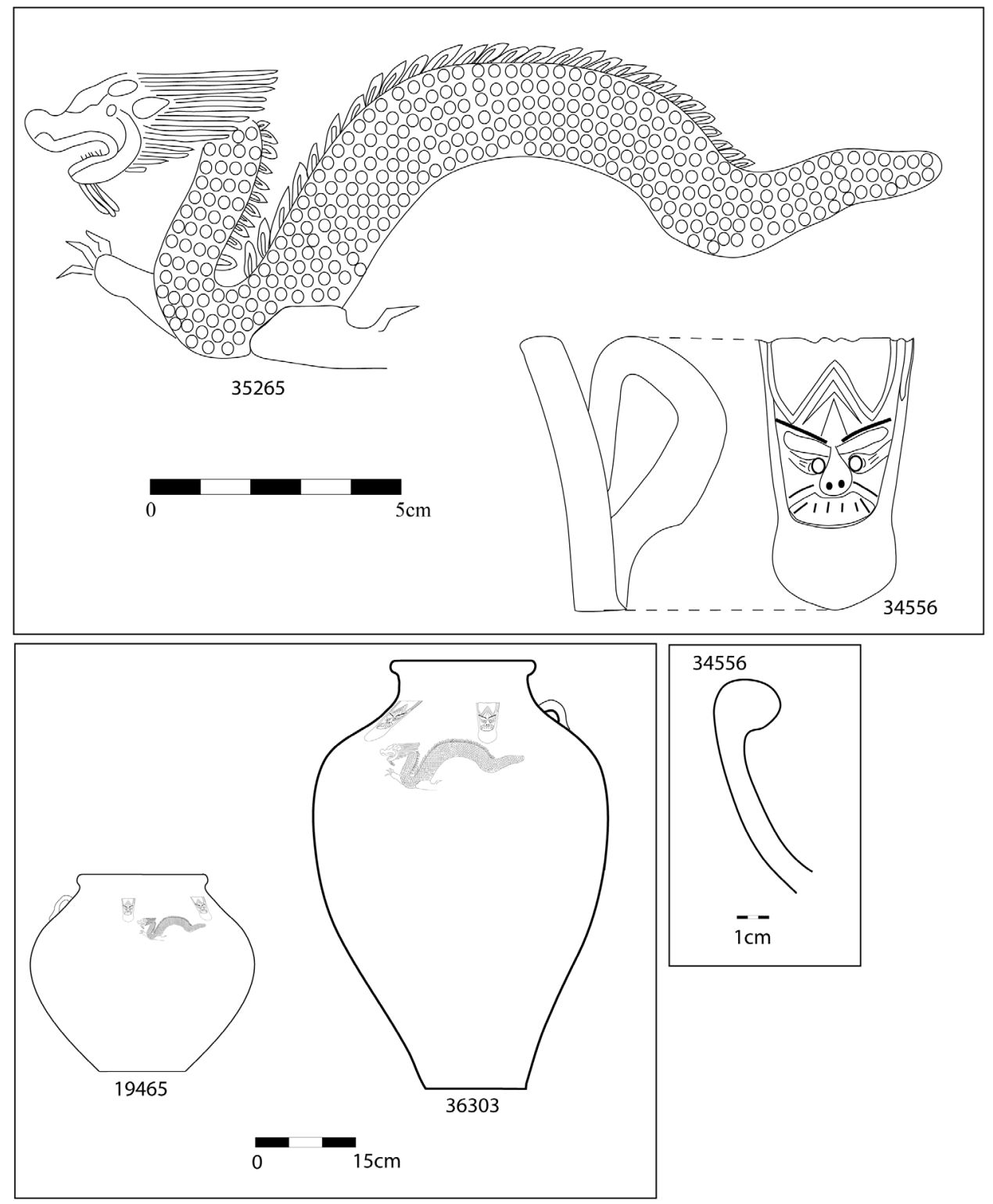

Fig. 1. Tradition 1 montage (primary motif, handle motif, and vessel form). Note that the neck of the dragon in \#34473 served as the lug handle.

They are covered with an olive to olive-brown glaze. Vessel shape is an inverted long convex cone with a wide, high shoulder and small base.

\section{Summary of Tradition 4 Jars}

The primary motifs of Tradition 4 jars are illustrated in Figure 4 and their production is summarized in Table 8 . These jars $(\mathrm{N}=10)$ are composed of a red paste of variable density and inclusions. They have everted rims with rectangular-shaped lips; vessel 

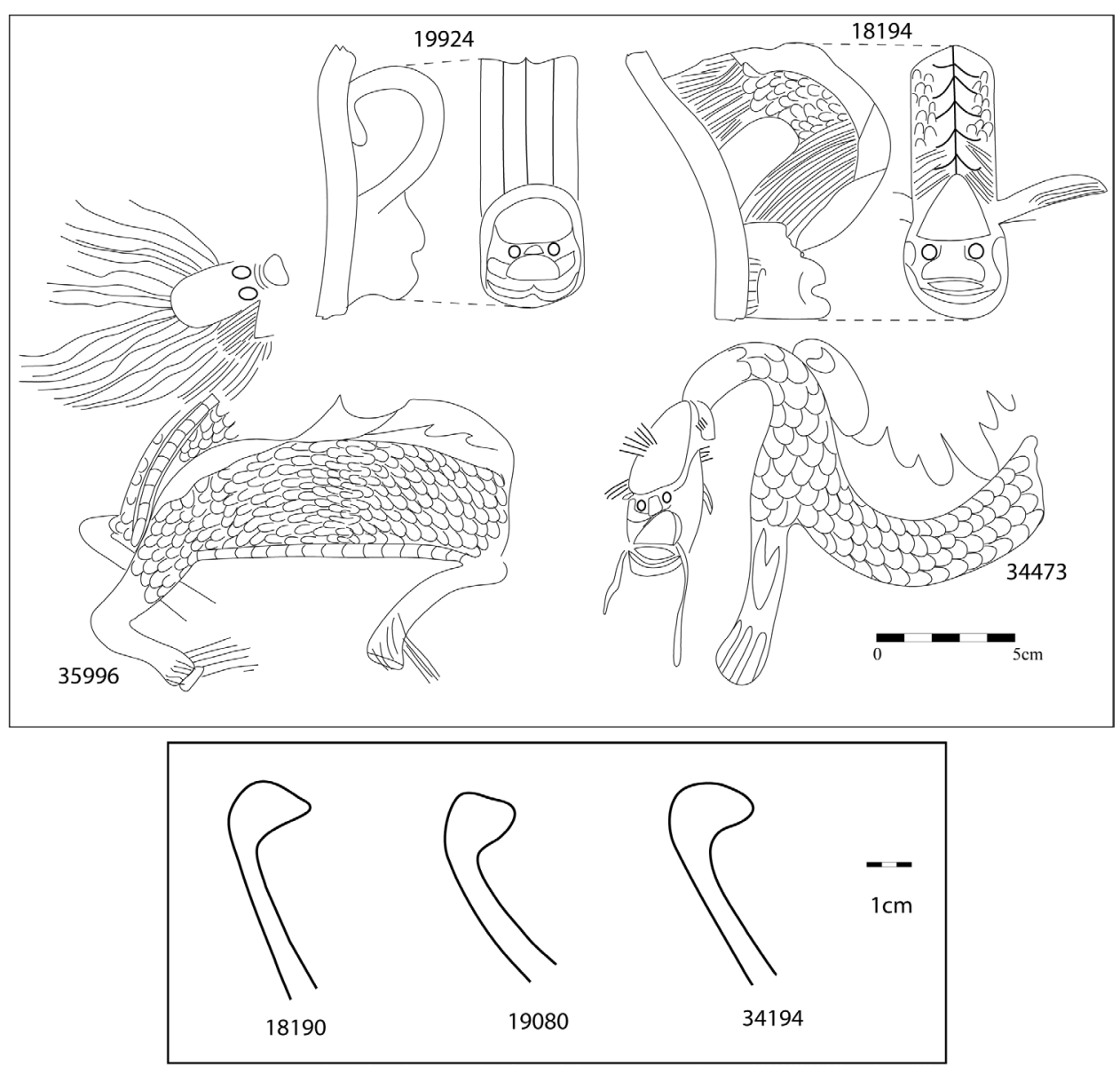

Fig. 2. Tradition 2 montage (primary motifs, handle motifs, and rim form).

thickness averages $6.8 \mathrm{~mm}$. There are two classes of visually similar primary motifs, representing dragons, fish, and flowers. The dragons are comparatively large, with whiskers and horns; the other motifs are very diverse and include symbols. The two classes are distinguished by different production techniques. One (4A) is a moldimpressed technique similar to Tradition 1, although the dragons are stylistically different and often impressed on the vessel body rather than the shoulder. The other (4B) is mold-attached, similar to Tradition 2 but without incised and impressed embellishments. 4A vessels sometimes have floral designs, whereas the $4 \mathrm{~B}$ vessels in the sample only have dragons. Both have horizontal strap handles with simple incised lines. They are covered with a dark yellow-brown glaze.

\section{DRAGON JAR PRODUCTION LOCALES}

The decorative variability discussed above revealed four very different traditions of pottery manufacture for the dragon jars in the Guthe Collection. To establish how these traditions were distributed in space (i.e., in discrete centers of production) and 


\begin{tabular}{|c|c|c|c|c|c|c|}
\hline 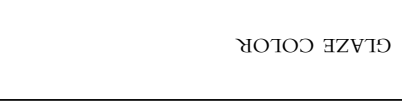 & 总 & 莺 & 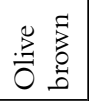 & 总葛 & 光言 & 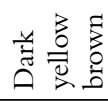 \\
\hline 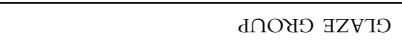 & | & | & | & - & - & - \\
\hline SNOISRTIDNI ALSVd & 恙 & 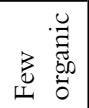 & 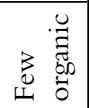 & 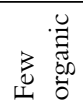 & 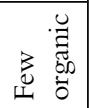 & 莺 \\
\hline XIISNGG GNY YOTOJ GLSVd & 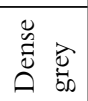 & 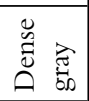 & 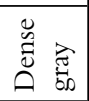 & 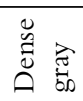 & 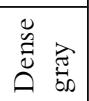 & 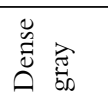 \\
\hline 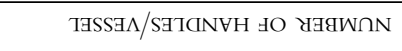 & 6 & 6 & | & I & I & I \\
\hline HILOW ヨาCINVH & $\begin{array}{l}\overline{5} \\
\stackrel{5}{0}\end{array}$ & $\begin{array}{l}\overrightarrow{5} \\
\stackrel{5}{0} \\
.\end{array}$ & $\begin{array}{l}7 \\
\overline{0} \\
0 \\
0\end{array}$ & $\begin{array}{l}7 \\
\overline{0} \\
0 \\
0\end{array}$ & $\begin{array}{l}7 \\
\overline{0} \\
\stackrel{0}{0}\end{array}$ & $\begin{array}{l}7 \\
\overline{0} \\
0\end{array}$ \\
\hline NOILVLNAIYO ヨ]CNVH & 苞 & 苞 & 苞 & 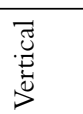 & 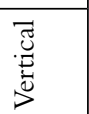 & 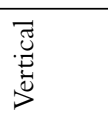 \\
\hline 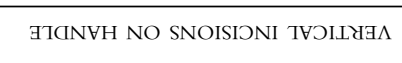 & $\ddot{*}$ & $\ddot{v}$ & $\ddot{v}$ & 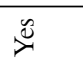 & $\stackrel{\vartheta}{\vartheta}$ & $\triangleq$ \\
\hline 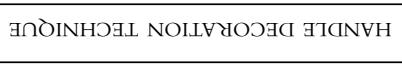 & $\bar{\Sigma}$ & $\bar{\Sigma}$ & $\bar{\Sigma}$ & $\bar{\Sigma}$ & $\bar{\Sigma}$ & $\bar{\Sigma}$ \\
\hline TASSA $\Lambda$ NO NOILYYOJAC GASIJNI & $\stackrel{\circ}{Z}$ & 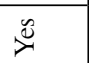 & $\stackrel{\circ}{Z}$ & $\stackrel{\circ}{Z}$ & $\stackrel{\circ}{Z}$ & $\stackrel{\circ}{Z}$ \\
\hline $\begin{array}{r}\text { NOILVכOT } \\
\text { (NOJVYA) HILOW } \text { RYVWIXd }\end{array}$ & 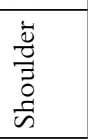 & $\begin{array}{l}\overrightarrow{0} \\
\frac{\overrightarrow{0}}{\vec{z}} \\
\frac{0}{n} \\
\frac{\pi}{n}\end{array}$ & 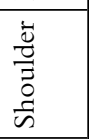 & 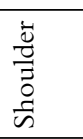 & $\begin{array}{l}\frac{\dot{v}}{0} \\
\frac{0}{3} \\
0 \\
\bar{\sigma}\end{array}$ & $\begin{array}{l}\vec{v} \\
\frac{\vec{v}}{\vec{z}} \\
\frac{0}{n} \\
\frac{\pi}{n}\end{array}$ \\
\hline $\begin{array}{l}\text { NOILVLNAIYO } \\
\text { (NOJVYA) HILOW } x \text { HYWIYd }\end{array}$ & 岕 & $\underset{\sqcup}{\stackrel{\Delta}{\leftrightarrows}}$ & 苟 & | & | & $\stackrel{\text { 岕 }}{\leftrightarrows}$ \\
\hline 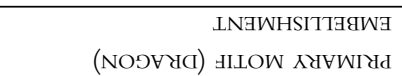 & $\bar{\Sigma}$ & $\bar{\Sigma}$ & $\bar{\Sigma}$ & $\bar{\Sigma}$ & $\dot{\Sigma}$ & $\bar{\Sigma}$ \\
\hline 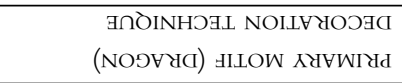 & $\bar{\Sigma}$ & $\bar{\Sigma}$ & $\bar{\Sigma}$ & $\bar{\Sigma}$ & $\dot{\Sigma}$ & $\bar{\Sigma}$ \\
\hline 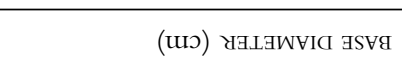 & $\begin{array}{l}\infty \\
\stackrel{\infty}{\sim}\end{array}$ & $\hat{\mathrm{d}}$ & | & $\begin{array}{l}\text { in } \\
\text { In }\end{array}$ & | & | \\
\hline 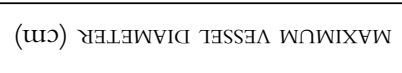 & $\dot{m}$ & $\stackrel{n}{n}$ & | & | & | & | \\
\hline 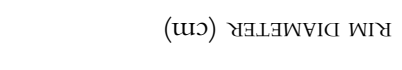 & in & $\bar{\sigma} \vec{\sim}$ & | & $\stackrel{\infty}{\sim} \stackrel{\infty}{\sim}$ & | & $\stackrel{\infty}{\sim} \stackrel{\infty}{\sim}$ \\
\hline (шо) цнэган таsรа $\Lambda$ & 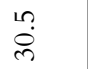 & $\ddot{n}$ & | & | & | & | \\
\hline (யuU) SSANYวIHL TaSSA $\Lambda$ & เ & 10 & in & in & $\stackrel{n}{r}$ & 6 \\
\hline aNYTSI & $\begin{array}{l}\overrightarrow{0} \\
\frac{7}{0} \\
0\end{array}$ & Uे & Uे & $\begin{array}{l}\vec{F} \overrightarrow{0} \\
\overrightarrow{0} \\
\overrightarrow{0} \\
0 \\
0\end{array}$ & 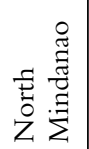 & 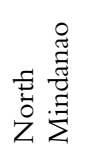 \\
\hline \# SWO & 1 & 1 & 1 & $\stackrel{\infty}{\sim}$ & $\tilde{\lambda}$ & I \\
\hline \# ヨН.ภつ & $\begin{array}{l}\stackrel{\vec{n}}{\stackrel{n}{n}} \\
\stackrel{n}{n}\end{array}$ & 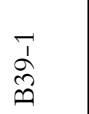 & 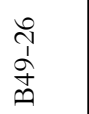 & $\stackrel{1}{\vec{\omega}} \stackrel{\text { m }}{m}$ & $\begin{array}{l}\overrightarrow{1} \\
\stackrel{\infty}{N} \\
\text { J }\end{array}$ & $\begin{array}{l}\hat{1} \\
\infty \\
\tilde{U}\end{array}$ \\
\hline 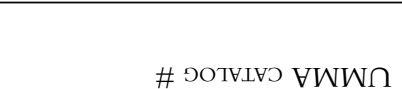 & $\begin{array}{l}n \\
0 \\
0 \\
0 \\
2\end{array}$ & 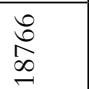 & $\begin{array}{l}\infty \\
\substack{\infty \\
\infty \\
\infty \\
1}\end{array}$ & 衷 & 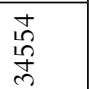 & 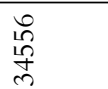 \\
\hline
\end{tabular}




\begin{tabular}{|c|c|c|c|c|c|}
\hline 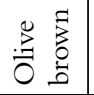 & 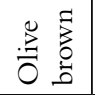 & 总产 & : & 莺害 & 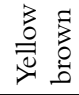 \\
\hline \begin{tabular}{l|l}
1 \\
\end{tabular} & 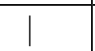 & 1 & - & 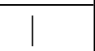 & $n$ \\
\hline 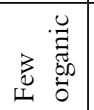 & 咅 & 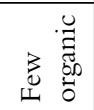 & 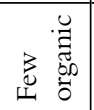 & 俤 & $\overleftarrow{z}$ \\
\hline 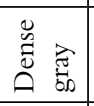 & 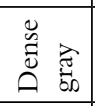 & 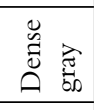 & 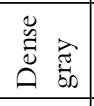 & 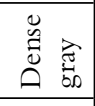 & 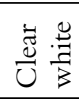 \\
\hline 1 & 1 & 0 & 1 & 0 & $m$ \\
\hline 胥 & $\begin{array}{l}\overline{\bar{E}} \\
\stackrel{\Delta}{ }\end{array}$ & $\begin{array}{l}\overline{\overrightarrow{5}} \\
\stackrel{\Lambda}{\Omega}\end{array}$ & $\begin{array}{l}\overline{\vec{\nu}} \\
\stackrel{0}{\Omega}\end{array}$ & $\begin{array}{l}\overline{\vec{\nu}} \\
\stackrel{0}{\Omega}\end{array}$ & 㽦 \\
\hline 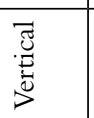 & 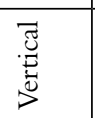 & 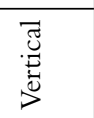 & 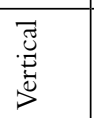 & 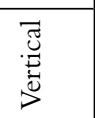 & 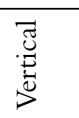 \\
\hline 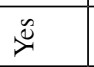 & : & $\check{\ddot{x}}$ & $\check{\varkappa}$ & $\tilde{x}$ & $\stackrel{0}{0}$ \\
\hline $\bar{\Sigma}$ & $\bar{\Sigma}$ & $\bar{\Sigma}$ & $\bar{\Sigma}$ & $\bar{\Sigma}$ & $\bar{\Sigma}$ \\
\hline ż & z̊ & $\ddot{\check{x}}$ & ż & ż & ż \\
\hline $\begin{array}{l}\frac{\tilde{v}}{\bar{v}} \\
\frac{\partial}{0} \\
\bar{n} \\
\end{array}$ & $\begin{array}{l}\vec{u} \\
\frac{\vec{v}}{\overrightarrow{0}} \\
\bar{d} \\
\bar{n}\end{array}$ & $\begin{array}{l}\vec{u} \\
\frac{\vec{v}}{\bar{a}} \\
\bar{m} \\
\bar{n}\end{array}$ & $\begin{array}{l}\vec{u} \\
\frac{\vec{\pi}}{\bar{\sigma}} \\
\bar{m}\end{array}$ & $\begin{array}{l}\vec{v} \\
\bar{z} \\
\bar{z} \\
\bar{\omega} \\
\end{array}$ & $\begin{array}{l}\vec{y} \\
\frac{0}{0} \\
\overline{0} \\
\bar{n}\end{array}$ \\
\hline 岕 & 莺 & 莺 & 1 & 岕 & 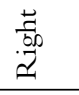 \\
\hline $\bar{\Sigma}$ & $\bar{\Sigma}$ & $\bar{\Sigma}$ & $\bar{\Sigma}$ & $\bar{\Sigma}$ & $\bar{\Sigma}$ \\
\hline $\bar{\Sigma}$ & $\bar{\Sigma}$ & $\bar{\Sigma}$ & $\bar{\Sigma}$ & $\bar{\Sigma}$ & $\bar{\Sigma}$ \\
\hline I & I & 1 & 1 & $\stackrel{0}{2}$ & in \\
\hline 1 & I & ले & 1 & 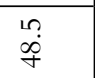 & $\stackrel{\infty}{\infty}$ \\
\hline 1 & $\pm \vec{i}$ & 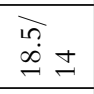 & 1 & 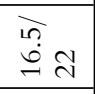 & in \\
\hline I & I & I & I & 6 & $\stackrel{\infty}{\llcorner}$ \\
\hline 0 & + & 0 & $\begin{array}{l}n \\
10 \\
10\end{array}$ & 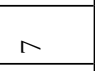 & 0 \\
\hline 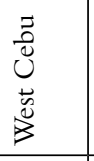 & $\begin{array}{l}\text { 音 } \\
\text { 砶 } \\
\text { U } \\
\end{array}$ & 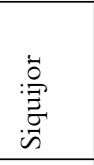 & 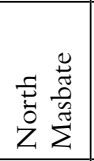 & 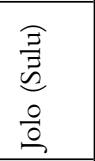 & 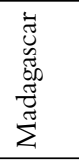 \\
\hline 1 & $\wedge$ & $\stackrel{\circ}{\circ}$ & ते & 1 & i \\
\hline ت্ & 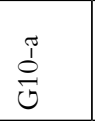 & $\begin{array}{l}\overline{1} \\
\infty \\
0 \\
0\end{array}$ & 音 & 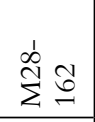 & 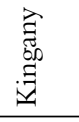 \\
\hline $\begin{array}{l}\stackrel{0}{\infty} \\
\stackrel{\infty}{\sim} \\
\stackrel{2}{\sim}\end{array}$ & 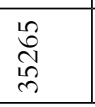 & 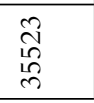 & हె & 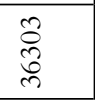 & I \\
\hline
\end{tabular}




\begin{tabular}{|c|c|c|c|c|c|c|c|}
\hline чотоЈ эZҰт९ & $\stackrel{气}{0}$ & $\stackrel{\Xi}{0}$ & 尝喜 & $\stackrel{亏}{0}$ & $\stackrel{\circ}{0}$ & $\stackrel{.}{0}$ & 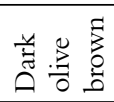 \\
\hline 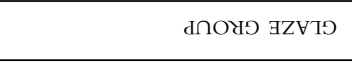 & $\frac{m}{d}$ & $\frac{n}{d}$ & 1 & 1 & I & 1 & I \\
\hline SNOISกTJNI ALSVd & 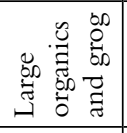 & 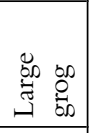 & 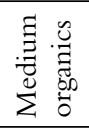 & 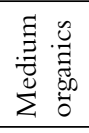 & 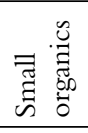 & 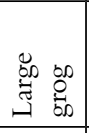 & 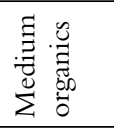 \\
\hline RLISNAC đNY YOTOO ALSVd & 空 & 苞 & 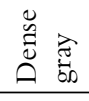 & 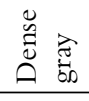 & 莺 & 总 & 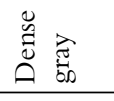 \\
\hline HILOW ヨTCNVH & $\stackrel{\infty}{\circ}$ & | & | & $\frac{\pi}{2}$ & | & $\stackrel{\infty}{\circ}$ & $\underset{0}{\leftrightarrows}$ \\
\hline NOILVLNAIYO ヨTCINYH & 莺 & | & | & 莺 & | & 莺 & : \\
\hline 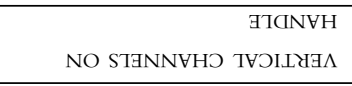 & - & | & I & | & | & - & | \\
\hline INAWHSITTAgWA ITCNHH & | & | & | & 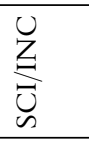 & | & | & 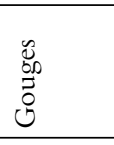 \\
\hline 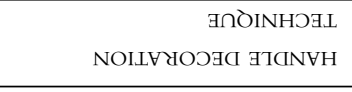 & $\$$ & | & | & 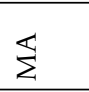 & | & $\$$ & $\$$ \\
\hline 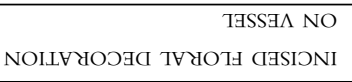 & | & | & | & | & | & | & 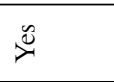 \\
\hline 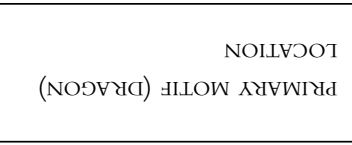 & $\begin{array}{l}\frac{\vec{v}}{0} \\
\frac{\overrightarrow{0}}{3} \\
\frac{\pi}{n}\end{array}$ & | & | & $\begin{array}{l}2 \\
\text { ते } \\
0 \\
0\end{array}$ & | & 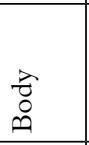 & | \\
\hline $\begin{array}{r}\text { NOILVLNAIYO } \\
\text { (NO5YYC) HILOW RYVWIYd } \\
\end{array}$ & 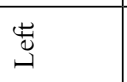 & | & 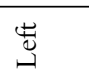 & | & | & | & | \\
\hline 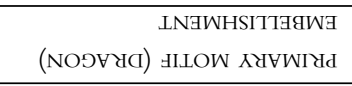 & l & $\overrightarrow{\mathrm{U}}$ & $\vec{U}$ & $\vec{U} Z$ & $\vec{U}$ & $\vec{U}$ & | \\
\hline 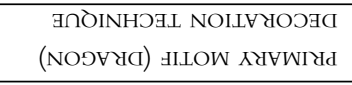 & 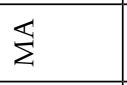 & 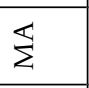 & 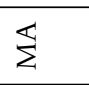 & 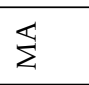 & 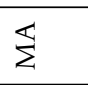 & $\sum$ & I \\
\hline 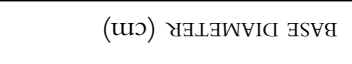 & | & | & | & | & | & | & | \\
\hline 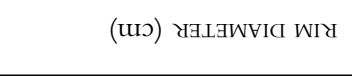 & $\stackrel{12}{2} \infty$ & $\lesssim \stackrel{i n g}{2}$ & I & | & | & I & | \\
\hline 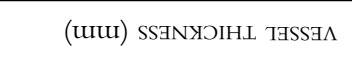 & $a$ & $\wedge$ & | & $a$ & 6 & 6 & | \\
\hline aNYTSI & 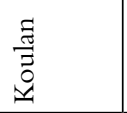 & 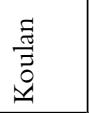 & 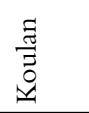 & 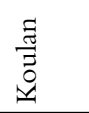 & 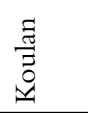 & 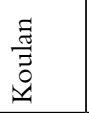 & $\begin{array}{l}\tilde{\Xi} \\
\stackrel{\Xi}{\Xi} \\
\qquad\end{array}$ \\
\hline \# SWO & $\stackrel{?}{+}$ & $\vec{m}$ & 1 & | & | & I & | \\
\hline \# มнเกอ & tे & 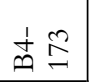 & t苞 & ๓ิ㇒ & $\frac{1}{ \pm} \stackrel{\infty}{=}$ & $\stackrel{1}{\infty} \stackrel{\infty}{\sim}$ & $\stackrel{1}{ \pm} \underset{\infty}{\infty}$ \\
\hline 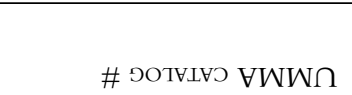 & $\begin{array}{l}\infty \\
+\infty \\
\infty \\
\infty\end{array}$ & $\underset{\infty}{\stackrel{\infty}{\infty}}$ & $\underset{\infty}{\stackrel{\infty}{\infty}}$ & 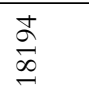 & 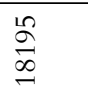 & $\stackrel{\substack{\infty \\
\infty}}{a}$ & 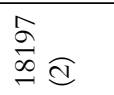 \\
\hline
\end{tabular}




\begin{tabular}{|c|c|c|c|c|c|c|c|}
\hline 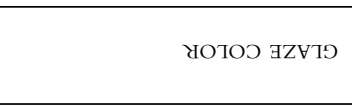 & 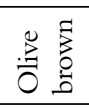 & 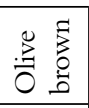 & $\stackrel{\circ}{\circ}$ & 党 & 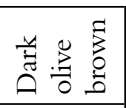 & 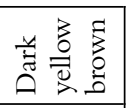 & 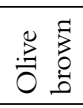 \\
\hline 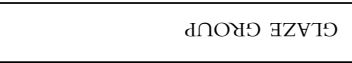 & 1 & 1 & 1 & $\frac{n}{d}$ & I & I & I \\
\hline SNOISOTJNI ILSVd & 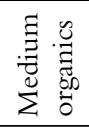 & 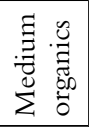 & 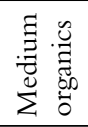 & 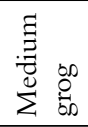 & 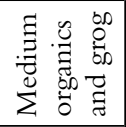 & 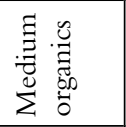 & 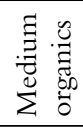 \\
\hline XLISNAC GNY YOTOJ ヨLSVd & 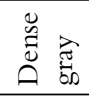 & 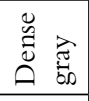 & 莺 & 莺 & 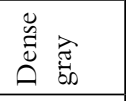 & 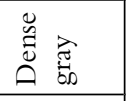 & 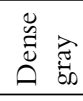 \\
\hline HILON घTCNVH & $\stackrel{\infty}{\circ}$ & $\stackrel{\infty}{\circ}$ & $\stackrel{\Xi}{\leftrightarrows}$ & | & | & I & : \\
\hline NOILVLNAIYO ヨTCNVH & 莺 & 苟 & 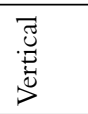 & 1 & | & I & 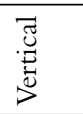 \\
\hline 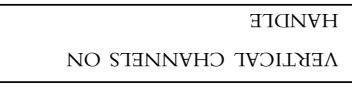 & $\sim$ & | & - & | & | & I & | \\
\hline INAWHSITTggWヨ घ7GINVH & | & | & $\begin{array}{l}0 \\
0 \\
0 \\
0 \\
0 \\
0\end{array}$ & I & | & | & $\begin{array}{l}0 \\
0 \\
0 \\
0 \\
0\end{array}$ \\
\hline $\begin{array}{r}\text { घกठัINHOAL } \\
\text { NOILYYOJAC ヨTGNYH } \\
\end{array}$ & $\$$ & $\S$ & 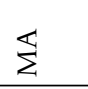 & 1 & | & | & $\$$ \\
\hline $\begin{array}{r}\text { TASSA NO NO } \\
\text { NOILVYOJaG TVYOTA GASIJNI } \\
\end{array}$ & $\stackrel{\varpi}{\approx}$ & 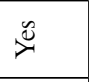 & | & $\triangleq$ & | & | & 1 \\
\hline 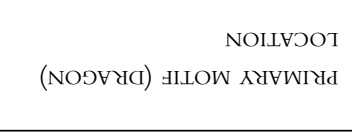 & | & | & 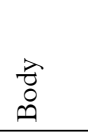 & | & | & | & $\begin{array}{l}\overrightarrow{2} \\
\frac{1}{0} \\
0 \\
\end{array}$ \\
\hline $\begin{array}{l}\text { NOILVLNAIYO } \\
\text { (NOJVYC) HILOW } \Lambda \text { YYWIYd }\end{array}$ & $\stackrel{\vec{\omega}}{\leftrightarrows}$ & | & | & | & | & 1 & $\underset{⿱ 中}{\leftrightarrows}$ \\
\hline 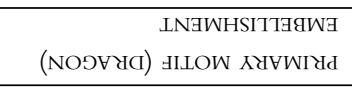 & $\overrightarrow{\vec{U}}$ & $\overrightarrow{\tilde{U}} \underline{Z}$ & $\overrightarrow{\mathrm{U}} \mathrm{U}$ & $\overrightarrow{\vec{U}}$ & 1 & $\overrightarrow{U_{n}}$ & $\overrightarrow{\vec{U}}$ \\
\hline 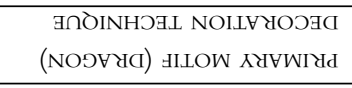 & 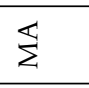 & 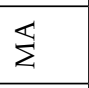 & 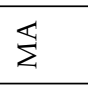 & 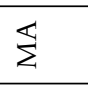 & | & 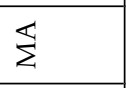 & 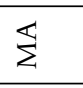 \\
\hline 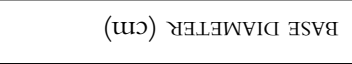 & | & | & 1 & 1 & 1 & 1 & | \\
\hline 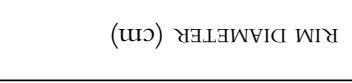 & 1 & | & 1 & $\sigma \approx$ & I & 1 & $\bar{\ominus} \bar{v}$ \\
\hline (uயu) SSANYOIHL TASSA $\Lambda$ & $\curvearrowright$ & $\begin{array}{l}10 \\
\infty\end{array}$ & $\infty$ & $\infty$ & $\wedge$ & $\underset{1}{2}$ & $\stackrel{\circ}{\sim}$ \\
\hline aNVTSI & 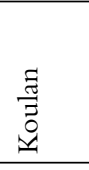 & 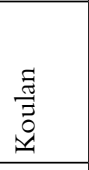 & 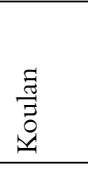 & 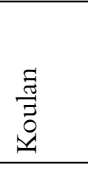 & 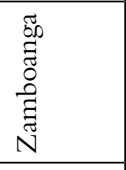 & $\overrightarrow{0}$ & 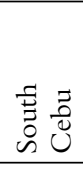 \\
\hline \# SWO & | & I & $\stackrel{\infty}{m}$ & $\approx$ & 1 & | & 1 \\
\hline \# घнเก९ & $\stackrel{+}{\infty} \underset{\infty}{-}$ & $\stackrel{1}{\infty} \stackrel{\infty}{=}$ & $\stackrel{1}{+} \stackrel{\infty}{\infty}$ & $\stackrel{+}{+} \stackrel{\infty}{\infty}$ & $\frac{1}{n} \simeq$ & $\frac{1}{\stackrel{1}{a}} a$ & $\stackrel{1}{\stackrel{1}{n}}$ \\
\hline \# כOTVLYว $\forall W W \cap$ & $\begin{array}{l}\infty \\
\stackrel{\infty}{\infty}\end{array}$ & $\begin{array}{l}8 \\
\stackrel{1}{1} \\
\infty \\
-\end{array}$ & $\begin{array}{l}\infty \\
\stackrel{N}{1} \\
\infty \\
-\end{array}$ & 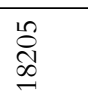 & $\begin{array}{l}+ \\
0 \\
⿱ \\
0 \\
-1\end{array}$ & \begin{tabular}{l}
0 \\
\multirow{2}{*}{} \\
2 \\
2
\end{tabular} & 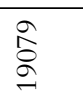 \\
\hline
\end{tabular}




\begin{tabular}{|c|c|c|c|c|c|}
\hline 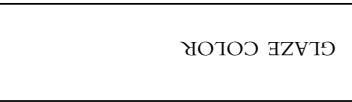 & 总言 & 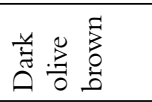 & 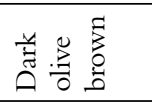 & 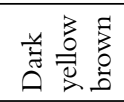 & 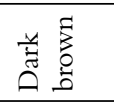 \\
\hline 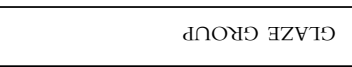 & $\frac{n}{d}$ & I & 1 & $\stackrel{m}{a}$ & 1 \\
\hline SNOISกTONI ALSHd & 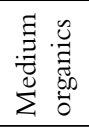 & 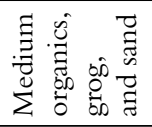 & 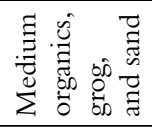 & 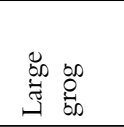 & 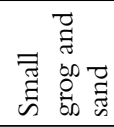 \\
\hline 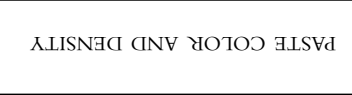 & 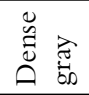 & 悹 & 氙 & त्ञ & 窇 \\
\hline HILOW घTCNVH & :̊̈ & $\stackrel{\infty}{\circ}$ & 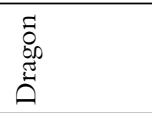 & $\stackrel{\infty}{\circ}$ & . \\
\hline NOILVLNGIYO ヨTCNVH & 莺 & 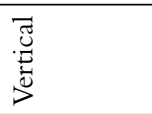 & 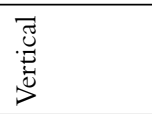 & 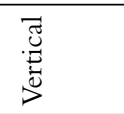 & 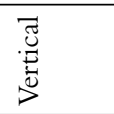 \\
\hline 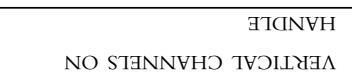 & - & N & | & - & | \\
\hline INAWHSITTAgWE ATCINVH & | & | & $\vec{心} \geqq$ & 1 & $\begin{array}{l}0 \\
0 \\
0 \\
0 \\
0 \\
0\end{array}$ \\
\hline 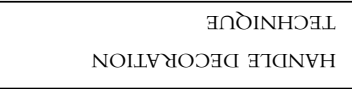 & $\begin{array}{l}\vec{Z} \\
\stackrel{\bar{I}}{I} \\
\end{array}$ & 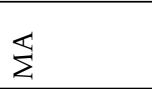 & $\sum$ & 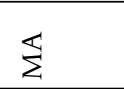 & $\S$ \\
\hline 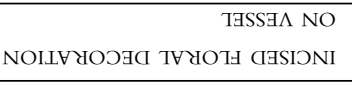 & 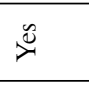 & | & | & | & $\ddot{\nu}$ \\
\hline 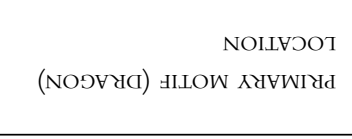 & | & $\begin{array}{l}x \\
0 \\
0 \\
0\end{array}$ & $\begin{array}{l}\frac{\overrightarrow{0}}{0} \\
\frac{\vec{J}}{0} \\
\frac{\vec{n}}{n}\end{array}$ & $\begin{array}{l}\vec{x} \\
0 \\
0 \\
\end{array}$ & l \\
\hline 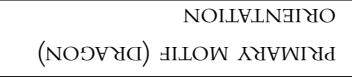 & | & | & | & | & 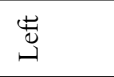 \\
\hline 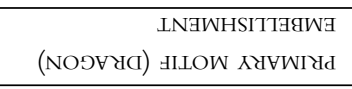 & $\vec{\circlearrowright}$ & $\dot{\circlearrowright}$ & $\vec{心} \tilde{Z}$ & $\vec{U}$ & $\overrightarrow{\tilde{U}}$ \\
\hline 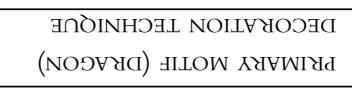 & 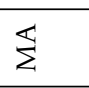 & 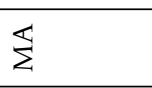 & 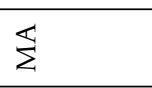 & $\S$ & $\S$ \\
\hline 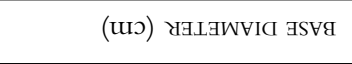 & | & 1 & 1 & | & | \\
\hline 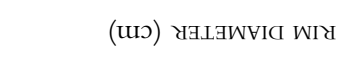 & $\stackrel{\infty}{n} \stackrel{\infty}{\sim}$ & 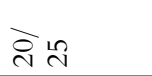 & I & I & $\stackrel{\operatorname{Ln}}{\sim} \stackrel{\infty}{\sim}$ \\
\hline (uய) SSANYOIHL TaSSA $\Lambda$ & $r$ & 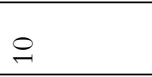 & 6 & 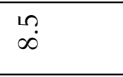 & $a$ \\
\hline GNVTSI & 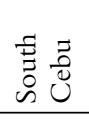 & ల్ల్ర & $\begin{array}{l}\tilde{\Xi} \\
\tilde{\Xi} \\
\tilde{n}\end{array}$ & $\begin{array}{l}\tilde{\Xi} \\
\tilde{\Xi} \\
\end{array}$ & $\begin{array}{l}\text { चี } \\
\text { हี } \\
\text { n }\end{array}$ \\
\hline \# SWO & $\stackrel{\infty}{i}$ & 斿 & $\stackrel{2}{q}$ & $a$ & $a$ \\
\hline \# घНเภऽ & $\stackrel{1}{\stackrel{n}{n}=}$ & 仓̊ & $\stackrel{b}{b} \stackrel{0}{0}$ & $\stackrel{b}{b} \infty$ & 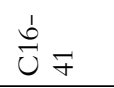 \\
\hline 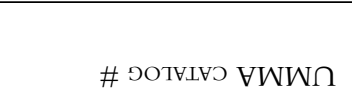 & $\begin{array}{l}0 \\
\infty \\
\vdots \\
\vdots\end{array}$ & $\stackrel{+}{\stackrel{+}{\alpha}}$ & $\begin{array}{l}\stackrel{\infty}{\infty} \\
\stackrel{\infty}{+}\end{array}$ & $\begin{array}{l}\vec{\sigma} \\
\vec{\sigma}\end{array}$ & $\underset{\vec{\sigma}}{\vec{\sigma}}$ \\
\hline
\end{tabular}




\begin{tabular}{|c|c|c|c|c|c|c|}
\hline 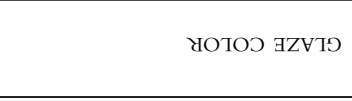 & 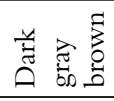 & 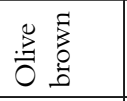 & $\stackrel{2}{0}$ & 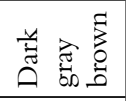 & $\stackrel{0}{0}$ & 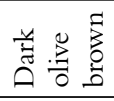 \\
\hline 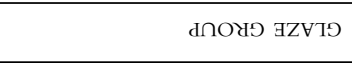 & I & 1 & 1 & $\frac{m}{d}$ & 1 & I \\
\hline SNOISRTJNI BLSVd & 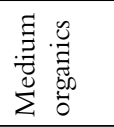 & 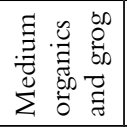 & 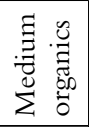 & 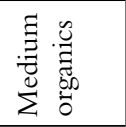 & 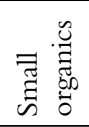 & 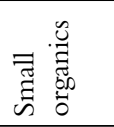 \\
\hline XLISNAC GNY YOTOO ALSVd & 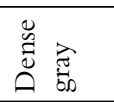 & 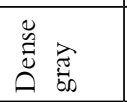 & 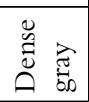 & 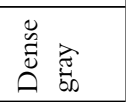 & 密 & 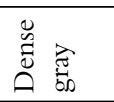 \\
\hline HILOW ヨาCNVH & .ేّ & $\stackrel{\Xi}{\leftrightarrows}$ & | & | & | & 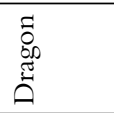 \\
\hline NOILVLNAIYO ヨTCNVH & 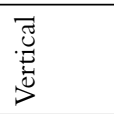 & 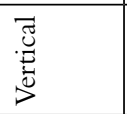 & | & 1 & | & 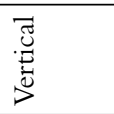 \\
\hline 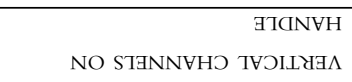 & | & I & I & I & | & 1 \\
\hline INGWHSITTAgWA ATCNVH & $\begin{array}{l}0 \\
0 \\
0 \\
0 \\
0 \\
0\end{array}$ & $\begin{array}{l}n \\
0 \\
0 \\
0 \\
0 \\
0\end{array}$ & | & | & | & $\vec{U}$ \\
\hline 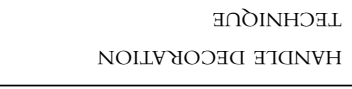 & 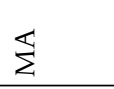 & $\sum$ & | & | & | & 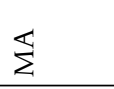 \\
\hline 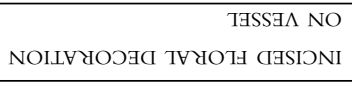 & | & 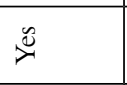 & 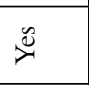 & | & | & | \\
\hline 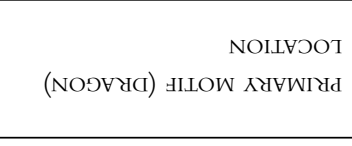 & 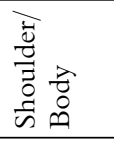 & $\begin{array}{l}\vec{v} \\
\frac{0}{z} \\
\vdots \\
\bar{n}\end{array}$ & $\begin{array}{l}\overrightarrow{2} \\
0 \\
0 \\
0\end{array}$ & $\begin{array}{l}\dot{\vec{g}} \\
\frac{0}{\overrightarrow{0}} \\
\frac{\pi}{n} \\
\end{array}$ & $\begin{array}{l}2 \\
0 \\
0 \\
0\end{array}$ & $\begin{array}{l}\frac{\dot{v}}{0} \\
\vec{z} \\
\frac{\pi}{n} \\
\end{array}$ \\
\hline $\begin{array}{l}\text { NOILVLNAIYO } \\
\text { (NOÐYYA) HILOW } \Lambda \text { YYWIYd }\end{array}$ & $\stackrel{\overrightarrow{0}}{\leftrightarrows}$ & | & | & $\stackrel{\vec{\omega}}{\leftrightarrows}$ & | & $\stackrel{\rightleftarrows}{\leftrightarrows}$ \\
\hline 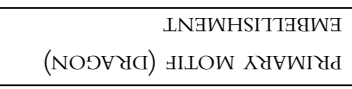 & $\overrightarrow{\mathrm{U}} \underline{\mathrm{n}}$ & | & $\vec{心}$ & $\overrightarrow{U_{Z}}$ & $\overrightarrow{\tilde{n}_{Z}}$ & $\overrightarrow{\vec{U}}$ \\
\hline 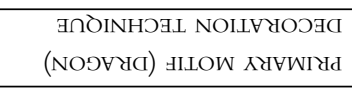 & 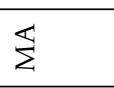 & 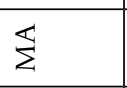 & 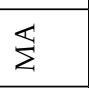 & $\S$ & $\S$ & $\gtreqless$ \\
\hline 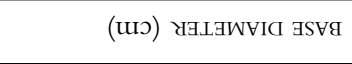 & | & | & | & | & | & $\stackrel{0}{\sim}$ \\
\hline 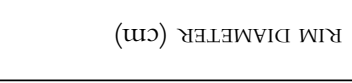 & | & in & | & $\underset{n}{\infty} \stackrel{\substack{n \\
-}}{2}$ & | & $\ddot{2}$ \\
\hline (UUu) SSANYOIHL TASSA $\Lambda$ & $=$ & $\wedge$ & $\wedge$ & $\stackrel{-}{\sim}$ & $a$ & | \\
\hline aNVTSI & $\underset{\overparen{\Xi}}{\tilde{\Xi}}$ & 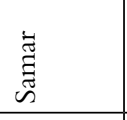 & $\begin{array}{l}\tilde{\Xi} \\
\text { Еี } \\
\end{array}$ & $\begin{array}{l}\text { चี } \\
\text { ڤี }\end{array}$ & $\begin{array}{l}\text { ⿹ี } \\
\text { हี } \\
\end{array}$ & 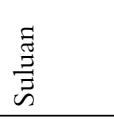 \\
\hline \# SWO & | & $\vec{子}$ & | & in & | & $\stackrel{n}{m}$ \\
\hline \# ヨНเภอ & $\stackrel{b}{\circlearrowright}$ ले & $\stackrel{b}{\breve{U}} \nsucceq$ & 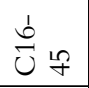 & $\dot{U}_{n}^{\prime}$ & $\frac{1}{\tilde{U}} \hat{n}$ & 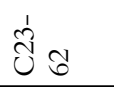 \\
\hline 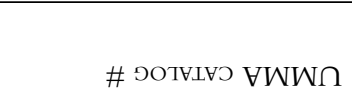 & 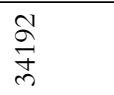 & 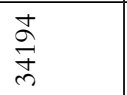 & $\begin{array}{l}\stackrel{1}{2} \\
\stackrel{2}{2} \\
\end{array}$ & $\begin{array}{l}F \\
\stackrel{+}{m} \\
\stackrel{m}{m}\end{array}$ & 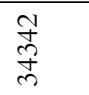 & $\stackrel{\substack{f \\
+}}{\stackrel{p}{m}}$ \\
\hline
\end{tabular}




\begin{tabular}{|c|c|c|c|c|c|c|c|}
\hline 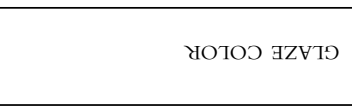 & 总 & 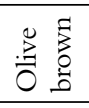 & 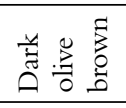 & 旁葛 & 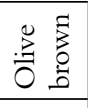 & 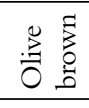 & 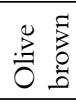 \\
\hline 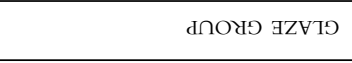 & $\frac{m}{d}$ & $\frac{m}{d}$ & I & $\stackrel{m}{n}$ & 1 & 1 & 1 \\
\hline SNOISกTONI BLSHd & 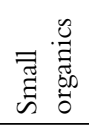 & 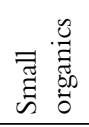 & 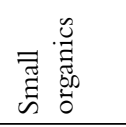 & 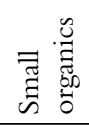 & 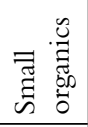 & 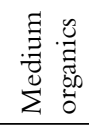 & 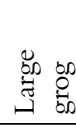 \\
\hline XLISNAC INY YOTOO ILSVd & 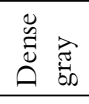 & 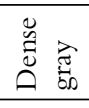 & 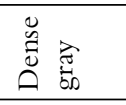 & 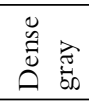 & 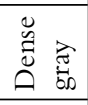 & 豞 & 空 \\
\hline HILOW ヨาđNVH & | & 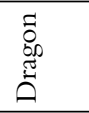 & | & $\ddot{\Xi}$ & | & $\stackrel{\infty}{\circ}$ & | \\
\hline NOILVLNGIYO ヨTCINYH & | & 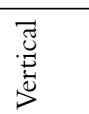 & | & 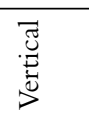 & | & 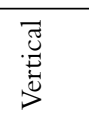 & | \\
\hline 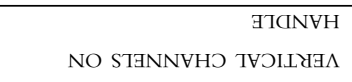 & | & | & | & - & | & $\mathrm{d}$ & | \\
\hline INAWHSITTAgWA ヨาINVH & | & $\vec{U} \geqq$ & | & | & 1 & | & 1 \\
\hline $\begin{array}{r}\text { घกÒINHOتL } \\
\text { NOILYYOJAG ヨTCNYH }\end{array}$ & | & 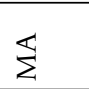 & | & $\begin{array}{l}\text { ت्ञ } \\
\text { ज्ञ } \\
\end{array}$ & | & 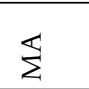 & 1 \\
\hline 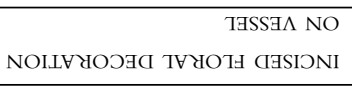 & | & | & | & 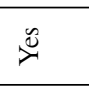 & | & 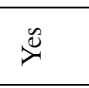 & | \\
\hline 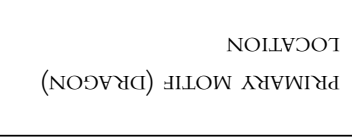 & $\begin{array}{l}\text { ते } \\
0 \\
0\end{array}$ & $\begin{array}{l}\frac{\vec{v}}{0} \\
\frac{0}{5} \\
\frac{0}{n} \\
\end{array}$ & | & | & | & 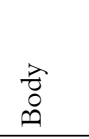 & | \\
\hline 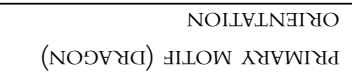 & | & 营 & | & $\stackrel{\text { 岕 }}{\omega}$ & | & 营 & 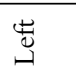 \\
\hline 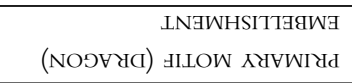 & $\vec{U}$ & $\vec{U}$ & $\vec{U}$ & $\vec{U}$ & $\vec{U}$ & $\vec{U}$ & $\overrightarrow{\tilde{U}}$ \\
\hline 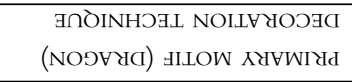 & $\sum$ & $\sum$ & 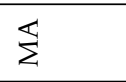 & $\sum$ & 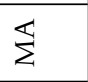 & $\sum$ & $\sum$ \\
\hline 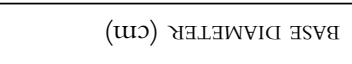 & | & | & | & | & $\stackrel{1}{\infty} \approx$ & | & | \\
\hline 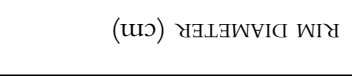 & 1 & $\underset{0}{\circ} \stackrel{10}{2}$ & | & $\exists \stackrel{i n}{I}$ & | & 1 & $\underset{ }{\stackrel{2}{2}}$ \\
\hline 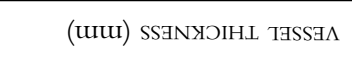 & $\curvearrowright$ & $a$ & $a$ & $\wedge$ & $\infty$ & $a$ & 6 \\
\hline aNYTSI & $\begin{array}{l}\ddot{\Xi} \\
\tilde{\Xi} \\
\tilde{n}\end{array}$ & $\begin{array}{l}\vec{E} \\
\vec{z} \\
0 \\
0 \\
0\end{array}$ & 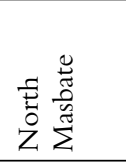 & 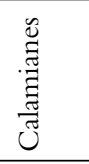 & 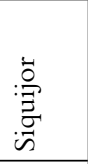 & 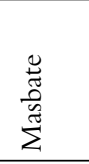 & 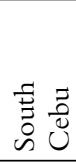 \\
\hline \# SWO & $\hat{m}$ & 导 & | & - & 1 & $m$ & | \\
\hline \# มНเภ๖ & 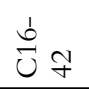 & $\stackrel{1}{\stackrel{J}{U}} a$ & 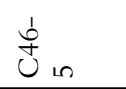 & 克o & b & $\stackrel{\dot{\prime}}{\Sigma} \bar{v}$ & $\sum^{b} \gamma$ \\
\hline \# ๑OTVLVO $\forall W W \cap$ & $\begin{array}{l}\stackrel{2}{a} \\
\stackrel{q}{+} \\
m\end{array}$ & $\begin{array}{l}\text { N̂ } \\
\text { o } \\
\text { ஸे }\end{array}$ & $\begin{array}{l} \\
\text { J } \\
b \\
c \\
m\end{array}$ & $\begin{array}{l}0 \\
\text { مn } \\
\infty \\
\infty \\
0 \\
\end{array}$ & 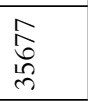 & $\begin{array}{l}\text { \& } \\
\text { مे } \\
\text { rn }\end{array}$ & 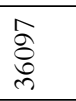 \\
\hline
\end{tabular}




\begin{tabular}{|c|c|c|c|}
\hline чотоЈ эZҰт९ & 莺 & 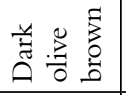 & $\stackrel{8}{0}$ \\
\hline 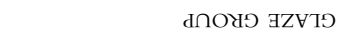 & | & | & | \\
\hline SNOISNTJNI ALSYd & 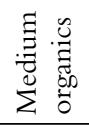 & 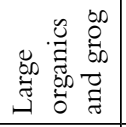 & 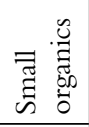 \\
\hline X.LISNAC GNY YOTOO ヨLSVd & 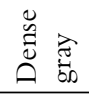 & 密 & 空 \\
\hline IILOW ヨTCINVH & | & 气̊̆ & $\stackrel{\infty}{\circ}$ \\
\hline NOILVLNAIYYO ヨTGNYH & | & 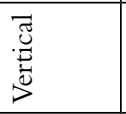 & 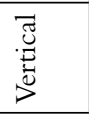 \\
\hline 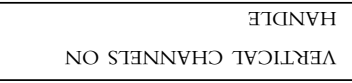 & | & - & | \\
\hline LNAWHSITTAgWA ヨ7CINYH & | & | & | \\
\hline 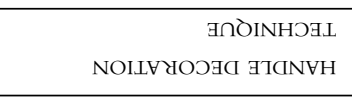 & | & $\begin{array}{l}\bar{Z} \\
\text { ज्ञ } \\
\text { I }\end{array}$ & 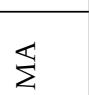 \\
\hline 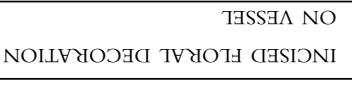 & | & | & | \\
\hline 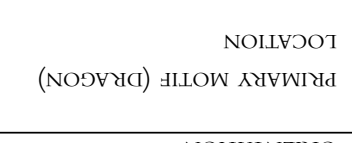 & | & 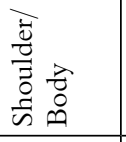 & $\begin{array}{l}2 \\
0 \\
0 \\
0\end{array}$ \\
\hline 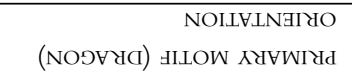 & 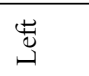 & | & | \\
\hline 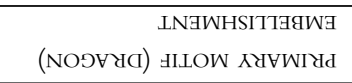 & $\vec{U}$ & $\vec{U}$ & $\vec{U}$ \\
\hline 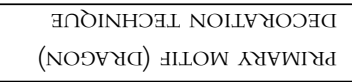 & 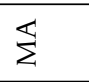 & $\sum$ & $\S$ \\
\hline 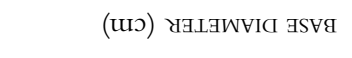 & | & | & | \\
\hline 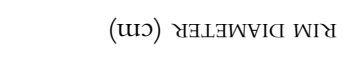 & | & | & 1 \\
\hline 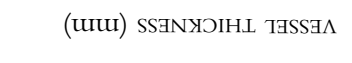 & $a$ & 6 & $a$ \\
\hline GNVTSI & 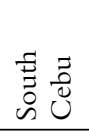 & 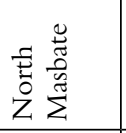 & 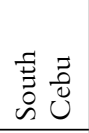 \\
\hline \# SWO & | & $\overleftarrow{Z}$ & 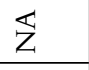 \\
\hline \# ヨНนภอ & $\stackrel{b}{\Sigma} \underset{+}{\infty}$ & $\stackrel{\dot{\prime}}{\Sigma} \infty$ & $\vec{I}$ \\
\hline \# ऽOTVLVכ $\forall W W \cap$ & \begin{tabular}{l}
$\infty$ \\
\multirow{2}{0}{} \\
0 \\
0 \\
0
\end{tabular} & 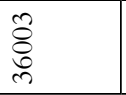 & | \\
\hline
\end{tabular}



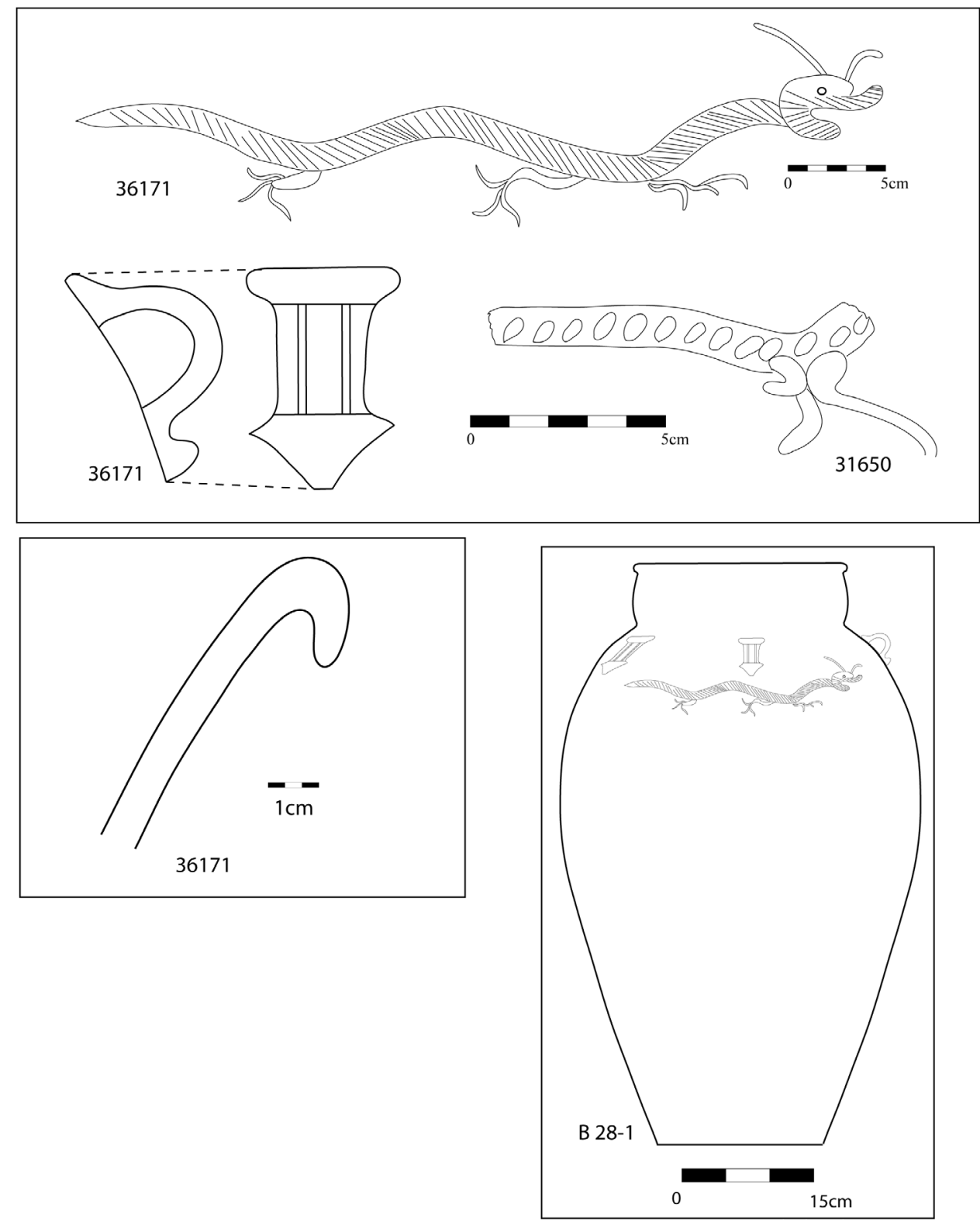

Fig. 3. Tradition 3 montage (primary motifs, handle motif, and vessel form).

time, it is necessary to explore variability of material procurement, paste preparation, and vessel formation. The four traditions are discussed in relation to evidence for different production locales in this section.

As Sinopoli and colleagues (2006) have discussed, INAA analyses conducted the University of Missouri Research Reactor (MURR) laboratory on 29 dragon jars (and 21 undecorated stoneware jars) identified four distinct compositional signatures for 


\begin{tabular}{|c|c|c|c|c|c|c|}
\hline 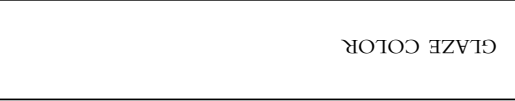 & 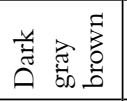 & 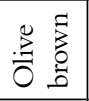 & $\stackrel{\frac{\pi}{3}}{0}$ & 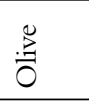 & 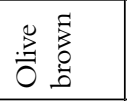 & $\stackrel{\frac{\pi}{0}}{0}$ \\
\hline 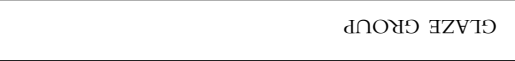 & $\frac{m}{d}$ & 1 & 1 & $\frac{m}{d}$ & | & 1 \\
\hline SNOISกTJNI ALSVd & 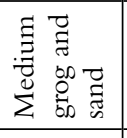 & 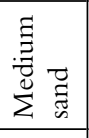 & 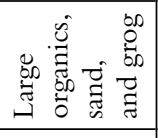 & 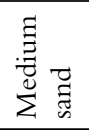 & 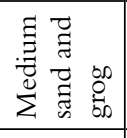 & 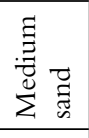 \\
\hline XIISNAC đNY YOTOつ ヨLSVd & 悹 & 密当 & 矛 & 氙 & 忿当 & 窇当 \\
\hline 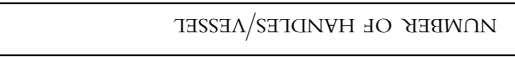 & I & 6 & I & I & 6 & 6 \\
\hline HILOW ヨТđNVH & $\begin{array}{l} \\
\check{Z} \\
z\end{array}$ & $\begin{array}{l}\check{0} \\
\text { Zे }\end{array}$ & $\begin{array}{l}\check{0} \\
\tilde{Z}\end{array}$ & $\begin{array}{l} \\
\tilde{Z} \\
z\end{array}$ & $\begin{array}{l} \\
\tilde{0} \\
z\end{array}$ & 苂 \\
\hline NOILVLNGIYO ヨTGNVH & : & : & | & : & 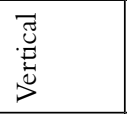 & : \\
\hline 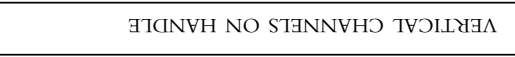 & I & $\mathrm{a}$ & I & I & $\mathrm{N}$ & $\mathrm{a}$ \\
\hline 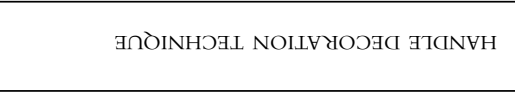 & $\begin{array}{l}\vec{E} \\
\text { I } \\
\end{array}$ & ت্: & | & I & $\begin{array}{l}\vec{\Xi} \\
\text { I } \\
\end{array}$ & $\begin{array}{l}\vec{Z} \\
\stackrel{\Xi}{I} \\
\end{array}$ \\
\hline 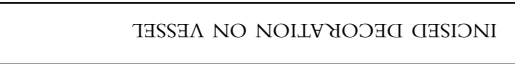 & I & $\triangleq$ & । & । & 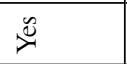 & $\stackrel{\pi}{*}$ \\
\hline 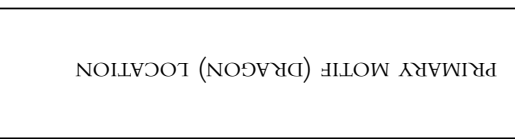 & $\begin{array}{l}\vec{v} \\
\frac{\vec{v}}{\vec{z}} \\
0 \\
\vec{n}\end{array}$ & $\begin{array}{l}\frac{\dot{v}}{0} \\
\frac{0}{3} \\
\frac{\pi}{n}\end{array}$ & $\begin{array}{l}\frac{\dot{v}}{0} \\
\frac{0}{0} \\
\frac{\pi}{n} \\
\end{array}$ & | & $\begin{array}{l}\frac{\dot{v}}{0} \\
\frac{0}{0} \\
\frac{\pi}{n}\end{array}$ & $\begin{array}{l}\frac{\dot{v}}{0} \\
\frac{0}{0} \\
\frac{1}{n}\end{array}$ \\
\hline 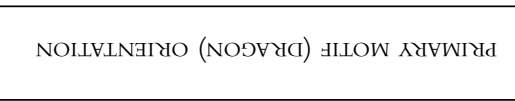 & | & 莺 & $\frac{\vec{n}}{20}$ & $\frac{\overrightarrow{5}}{20}$ & $\frac{1}{60}$ & $\frac{\vec{c}}{.00}$ \\
\hline 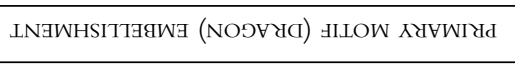 & I & 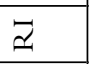 & 1 & $\widetilde{Z}$ & $\widetilde{Z}$ & $\widetilde{\simeq}$ \\
\hline 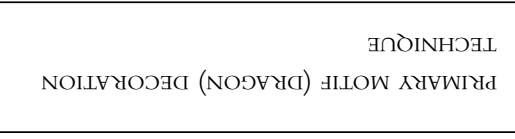 & 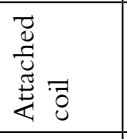 & 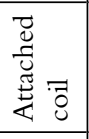 & 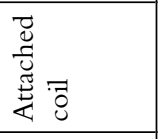 & 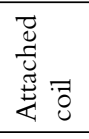 & 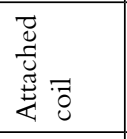 & 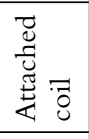 \\
\hline 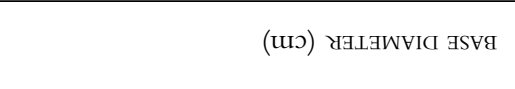 & | & $\stackrel{1}{\infty}$ & | & I & $\stackrel{1}{2}$ & $\stackrel{1}{2} \stackrel{2}{2}$ \\
\hline 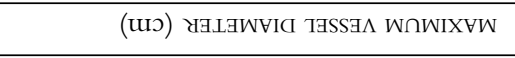 & I & ले & I & I & q & 守 \\
\hline 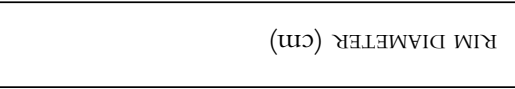 & | & 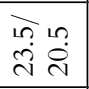 & | & | & 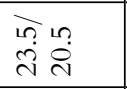 & 코 \\
\hline 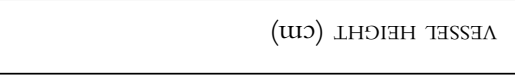 & | & 3 & | & | & 8 & $\stackrel{n}{i}$ \\
\hline 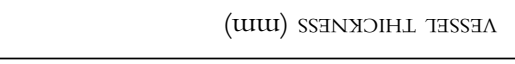 & ஸै & $\bullet$ & 10 & 0 & in & $\wedge$ \\
\hline aNVTSI & 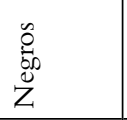 & 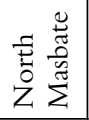 & $\vec{z}$ & 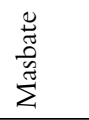 & $\begin{array}{l}\overrightarrow{0} \\
\stackrel{0}{0} \\
\overrightarrow{0}\end{array}$ & $\stackrel{0}{0}$ \\
\hline \# SWO & $\exists$ & 1 & I & $\infty$ & & 1 \\
\hline \# ヨН.ก९ & $\frac{1}{\infty} \stackrel{0}{=}$ & $\begin{array}{l}\dot{1} \\
\stackrel{N}{a}- \\
\infty\end{array}$ & $\frac{1}{\partial} \stackrel{d}{a}$ & 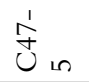 & 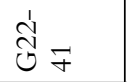 & 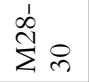 \\
\hline \# ગOTVLVつ VWW & 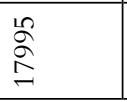 & 1 & $\begin{array}{l}\text { ì } \\
\infty \\
\infty \\
\infty\end{array}$ & 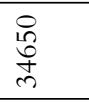 & 1 & 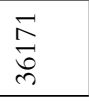 \\
\hline
\end{tabular}



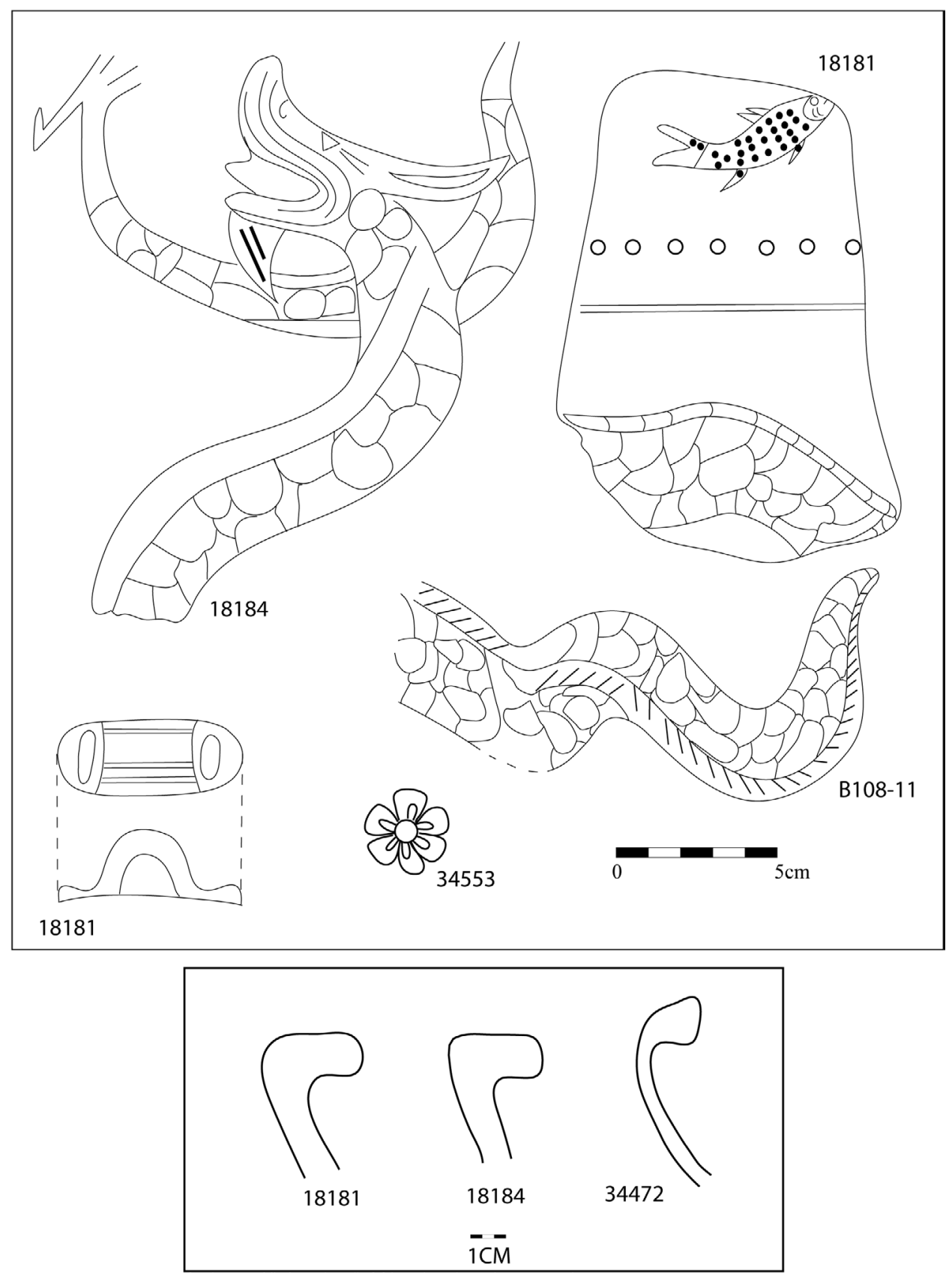

Fig. 4. Tradition 4 montage (primary motifs, handle motif, and rim form).

the traditions describe above. The results of principal component analyses suggest that vessels from Tradition 1 are the most distantly related to the other three groups, Tradition 2 and Tradition 3 are compositionally very similar, and Tradition 4 is distinct, but more similar to 2 and 3 than to 1 . 
Glaze analyses were performed both visually on 63 vessels and by compositional methods (LA-ICP-MS) on 20 vessels (Sinopoli et al. 2006). Compositional analyses reveal three groups, Tradition 1 and Tradition 4 are discrete, while Traditions 2 and 3 share a single glaze recipe. In principal component analyses, the composition of the glaze in Tradition 1 showed similarities to some elements of 2 and 3, as did other elements of the Tradition 4 glaze. Vessels from Traditions 1, 2, and 3 have glazes that are olive-brown, whereas Tradition 4 produced vessels that are dark yellow brown. This variation is to be expected, since Tradition 4 vessels have a red paste, while all the others use a gray paste (the glazes are semitranslucent). Since glaze recipes can be traded widely, the degree of variation between the traditions is difficult to discern. However, the differences between Tradition 1 and Traditions 2 and 3, despite their similar colors, suggest that these vessels were part of different production regimes.

Two general groupings can be made based on paste density and inclusions. Vessels from Tradition 1 have a homogeneous, dense, gray paste with few and small inclusions and vacua. Products of Traditions 2, 3, and 4 exhibit more heterogeneity within each group, ranging from dense to coarse pastes with many diverse large inclusions (i.e., sand, grog, organics).

Each vessel tradition has unique rim shapes. Rim diameter is fairly consistent across and between groups, despite the two size classes found in Tradition 1. Two groups in vessel thickness can be discerned, and cluster by decoration method. Vessels with mold-impressed and applied-coil dragons are thinner, and mold-applied decorated vessels are thicker. For example, Traditions 1 and 3 produce thinner vessels averaging $5.6 \mathrm{~mm}$ and $6 \mathrm{~mm}$. Traditions 4 and 2 are thicker vessels at $6.8 \mathrm{~mm}$ and $8.2 \mathrm{~mm}$, respectively. However, when Tradition 4 is divided into Traditions $4 \mathrm{~A}$ and $4 \mathrm{~B}$ wares, a clearer pattern emerges. Tradition $4 \mathrm{~A}$, the red ware with mold-impressed decorations, shows an average thickness of $6.3 \mathrm{~mm}$, whereas 4B, the red ware with mold-applied decorations, shows an average thickness of $8 \mathrm{~mm}$.

\section{TEMPORAL AND SPATIAL LOCALIZATION OF DRAGON JAR PRODUCTION}

As reviewed above and presented in detail in Sinopoli et al. (2006), compositional and other technological evidence indicate that three production centers were responsible for the four production traditions identified in the Guthe Collection. Enough information exists to suggest that Traditions 2 and 3 were produced at the same or very closely situated production locales $(\mathrm{B} / \mathrm{C})$. Tradition 4 should be considered as a unique production locale (D), and the vessels from Tradition 1 are the product of a strikingly different production locale (A) from the others. In the remainder of this article, I seek to explore more fully the available information on the chronology and locations of these centers and associated production traditions. In the absence of extensive research on or publication of relevant kiln sites, I draw upon a broad range of archaeological and textual research.

Information on production regions, use-life and dating for each of the production traditions can be divided into several classes of data. First, there is a growing literature on stylistic traits, motif characteristics, and the form of ceramics during the second millennium A.D. trade in Southeast Asia. Second, there is an ever-increasing body of data from the archaeological excavation or salvage of shipwrecks in the region. The wreck location and contents often provide clues as to ports of origin, routes, and destination, detailed information on the general assemblage of materials that were on 


\begin{tabular}{|c|c|c|c|c|}
\hline 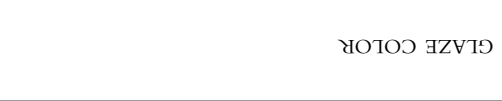 & 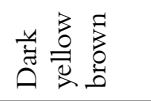 & 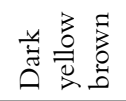 & 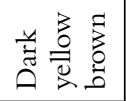 & 总葛 \\
\hline 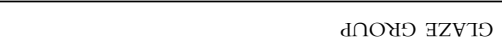 & + & | & I & I \\
\hline SNOISOTONI BLSVd & 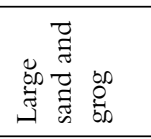 & 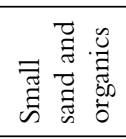 & $\begin{array}{l}3 \\
\text { 勇 } \\
\end{array}$ & 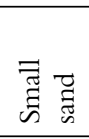 \\
\hline X.LISNAC INY YOTOJ GLSVD & 芯 & $\overrightarrow{0}$ & 远 & $\sum_{\bar{D}}^{2} \overline{0}$ \\
\hline HILOW ヨTGNVH & Zัँ & そ̆ & $\begin{array}{l}\text { İ } \\
\text { Ż }\end{array}$ & Z̃ \\
\hline NOILVINAIYO ヨTCINVH & 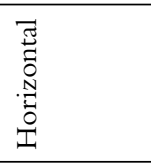 & 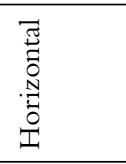 & 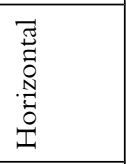 & 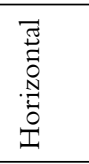 \\
\hline ITGNVH NO SNOISIONI TVLNOZIYOH & $n$ & $n$ & + & z \\
\hline ヨกठัINHOAL NOILYYOOتG ヨTGNYH & $\begin{array}{l}\vec{\Xi} \\
\stackrel{\Xi}{I}\end{array}$ & $\begin{array}{l}\vec{\Xi} \\
\vec{I} \\
\vec{I}\end{array}$ & $\begin{array}{l}\overrightarrow{\mathbb{I}} \\
\text { İ }\end{array}$ & $\begin{array}{l}\vec{Z} \\
\mathbb{I} \\
\widetilde{I}\end{array}$ \\
\hline TASSA $\Lambda$ NO NOILYYOOتA GASIJNI & $\stackrel{\circ}{Z}$ & Z & $\stackrel{\circ}{Z}$ & Z \\
\hline 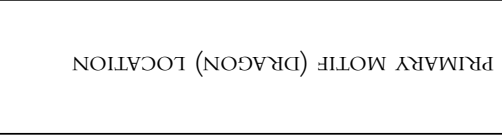 & $\begin{array}{l}\vec{x} \\
0 \\
0 \\
0\end{array}$ & 1 & $\begin{array}{l}\frac{\dot{v}}{0} \\
\frac{0}{3} \\
\frac{1}{n}\end{array}$ & $\begin{array}{l}\overrightarrow{0} \\
\dot{0} \\
0\end{array}$ \\
\hline 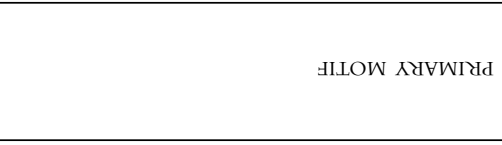 & 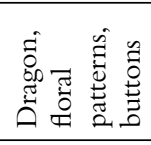 & 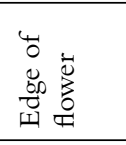 & 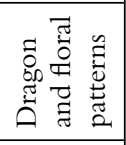 & 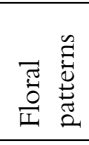 \\
\hline 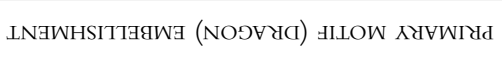 & $\bar{\Sigma}$ & $\bar{\Sigma}$ & $\bar{\Sigma}$ & $\bar{\Sigma}$ \\
\hline 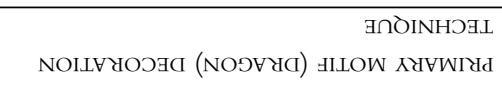 & $\bar{\Sigma}$ & $\xi$ & $\bar{\Sigma}$ & $\bar{\Sigma}$ \\
\hline 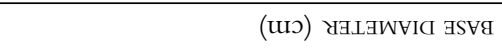 & | & I & | & I \\
\hline 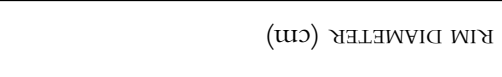 & $\stackrel{i n}{\infty} \stackrel{i n}{\sim}$ & 1 & I & 1 \\
\hline (UuU) SSANYOIHL TASSA $\Lambda$ & $\vec{\pi}$ & ○ & $\wedge$ & 6 \\
\hline INVIXVA & $\Varangle$ & $\Varangle$ & $\varangle$ & $\Varangle$ \\
\hline aNVTSI & 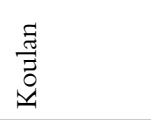 & 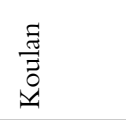 & 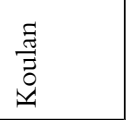 & 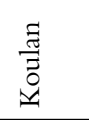 \\
\hline \# SWO & ๙ิ & I & I & I \\
\hline \# มн.กค & $\frac{1}{t} \underset{0}{0}$ & $+\frac{1}{\infty} \stackrel{1}{0}$ & $+\frac{1}{\infty} \stackrel{0}{\circ}$ & $+\frac{1}{\infty} \stackrel{\infty}{0}$ \\
\hline 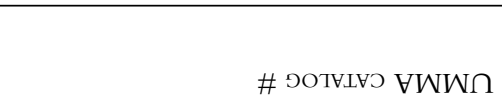 & $\underset{\infty}{\infty}$ & $\begin{array}{l}\infty \\
\infty \\
\infty \\
\infty\end{array}$ & $\begin{array}{l}\infty \\
\infty \\
\infty\end{array}$ & $\begin{array}{l}10 \\
\infty \\
\infty \\
\infty\end{array}$ \\
\hline
\end{tabular}




\begin{tabular}{|c|c|c|c|c|c|}
\hline 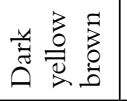 & 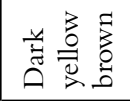 & 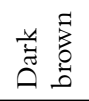 & 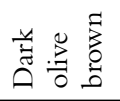 & 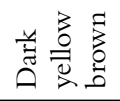 & 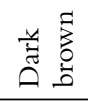 \\
\hline+ & + & 1 & | & + & + \\
\hline 虔 & 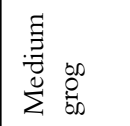 & 㤐 & 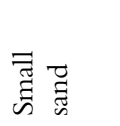 & 㤐 & 茎 \\
\hline 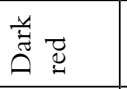 & 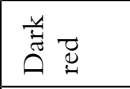 & 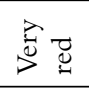 & ت્ّ & 己. & 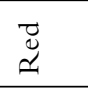 \\
\hline 1 & 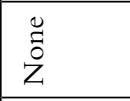 & 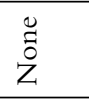 & 1 & $\begin{array}{l} \\
\tilde{Z}\end{array}$ & 1 \\
\hline I & 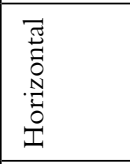 & 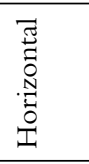 & 1 & 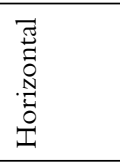 & 1 \\
\hline 1 & $\stackrel{\circ}{Z}$ & 1 & 1 & $m$ & 1 \\
\hline 1 & $\begin{array}{l}\vec{J} \\
\text { जี } \\
\text { I }\end{array}$ & $\begin{array}{l}\vec{T} \\
\text { Iี } \\
\text { T }\end{array}$ & I & $\begin{array}{l}\vec{T} \\
\mathbb{I} \\
\end{array}$ & 1 \\
\hline$\stackrel{\circ}{Z}$ & Z & Z & Ż & Z & $\stackrel{\circ}{Z}$ \\
\hline $\begin{array}{l}\text { ते } \\
0 \\
0\end{array}$ & 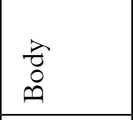 & I & 1 & $\begin{array}{l}x \\
0 \\
0 \\
0\end{array}$ & $\begin{array}{l}\overrightarrow{2} \\
0 \\
0 \\
0\end{array}$ \\
\hline 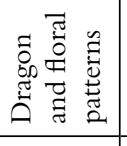 & 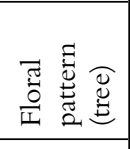 & 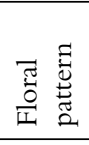 & 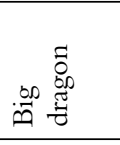 & 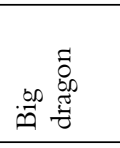 & 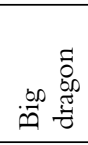 \\
\hline $\bar{\Sigma}$ & $\bar{\Sigma}$ & $\bar{\Sigma}$ & $\sum$ & $\sum$ & $\sum$ \\
\hline $\bar{\Sigma}$ & $\Sigma$ & $\Sigma$ & 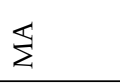 & 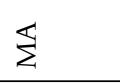 & $\S$ \\
\hline$\stackrel{\text { In }}{\mathrm{n}}$ & 1 & 1 & 1 & 1 & 1 \\
\hline 1 & $\stackrel{\ominus}{ \pm}$ & I & I & 1 & 1 \\
\hline 6 & 0 & 0 & $\infty$ & $\infty$ & $\infty$ \\
\hline$\varangle$ & $\varangle$ & 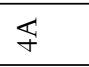 & $q$ & $\stackrel{\wp}{F}$ & 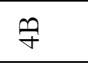 \\
\hline 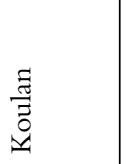 & $\frac{\Xi}{5}$ & $\begin{array}{l}\widetilde{50} \\
\text { ह๊ } \\
\text { Dे } \\
\text { है } \\
\text { N }\end{array}$ & $\begin{array}{l}\overrightarrow{0} \\
\text { ¿ } \\
0 \\
0\end{array}$ & 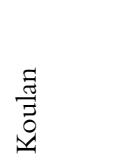 & 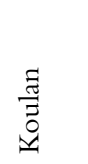 \\
\hline 字 & $\stackrel{0}{\circ}$ & 1 & 1 & ले & P \\
\hline $\begin{array}{l}0 \\
i \\
+1 \\
\text { in }\end{array}$ & 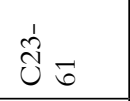 & $\hat{U}_{n}^{\prime}$ & 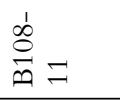 & $+\frac{1}{0}$ & $\begin{array}{l}\infty \\
⿱ 亠 䒑 \\
+1\end{array}$ \\
\hline $\begin{array}{l}\text { f } \\
\infty \\
\infty\end{array}$ & 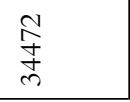 & 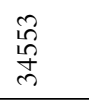 & | & $\begin{array}{l}+ \\
\infty \\
\infty \\
-1\end{array}$ & $\begin{array}{l}n \\
\stackrel{2}{8} \\
\infty\end{array}$ \\
\hline
\end{tabular}


board, and sometimes a precise date based on the records of the ship's loss. Third, there are the emerging results of archaeological investigations of kiln sites in Central Viet Nam by Vietnamese and Japanese researchers. These kilns are believed to be associated with Vijaya, one of the central states of Champa.

\section{Production Locale A (Tradition 1)}

All standard Tradition 1 jars were produced in a single production locale.

No shipwreck information is yet available for production Tradition 1 . Valdes et al. (1992:114-115, plates 39-40) attribute similar vessels to southern Chinese kilns near Quanzhou port in Guangdong Province, dating to the thirteenth and fourteenth centuries. Harrisson (1986) suggests a similar provenience and date. Similar jars recovered from Malaysia are dated to the end of the thirteenth century to the fourteenth and fifteenth centuries; in addition, Tradition 1 handles have been found in a context dating to the Song/Yuan period (Lam et al. 1985:111-112, plates 230-234, 236). Since the Pandanan and San Diego shipwrecks did not contain any vessels with Tradition 1 primary motifs, and coupled with the above known attributions, it is possible that Tradition 1 vessels were produced before the mid-fifteenth century.

Technologically, evidence for the organization of production of this group matches well with a Chinese origin, with standardized production (exterior and interior homogeneity) similar to that described in other archaeological artifacts produced by Late Song, Yuan, and Early Ming period Chinese artisans and found in the Philippines. Temporally, this group matches well with known information on the Late Song, Yuan, and Early Ming dynasties' direct and intense trade in the Philippines between the fourteenth and fifteenth centuries, and likely was produced during this period. No similar vessels are known from Vietnamese sites.

\section{Production Locale B/C (Traditions 2 and 3)}

Jars from Tradition 2, Tradition 3, and the Kingany vessel (alternative Tradition 1) were all produced at either the same or very closely situated production locales at different times. Collectively, production at locales $\mathrm{B} / \mathrm{C}$ spans the entire temporal period under discussion.

Tradition 2 vessels are known from several shipwreck sites, dating from the mid1400 s to 1600 . All of the dragon jar vessels reported from the A.D. 1600 San Diego shipwreck (Desroches et al. 1996; Valdes and Alba 1993) belong to Tradition 2. Published jars from the San Diego are identical to those found in the Guthe Collection in form, decoration, handle techniques, and intra-group diversity. The broad diversity present in the San Diego jars suggests that the intra-tradition variability in form was synchronic.

Jars of this tradition were also recovered from a fifteenth-century shipwreck off Marinduque; they are attributed to the southeast Chinese port of Zhangzhou in Fujian Province (Quimpo 1982:33-48, plates from pages 35, 46, and 47). A midfifteenth-century shipwreck off Pandanan Island contains dragon jars with Tradition 2 primary motifs (Loviny 1996:39, 54). This ship is believed to have sailed from central Viet Nam (Diem 1999, 2004). In this case, the dragon looks visually similar, with impressed scale embellishments; however, this dragon jar has a Tradition 1 mold- 
impressed handle. In our sample of 35 jars in Tradition 2 , no vessels had a handle resembling those from Tradition 1.

Several scholars have proposed chronological and provenience attributions for Tradition 2 jars. According to Valdes et al. (1992), the jars likely derive from the Guangdong region in China and date to the sixteenth century. However, Harrisson (1986) dates them to fifteenth-sixteenth-century Viet Nam, and John Guy (1986:111, plate 105) attributes Tradition 2 dragon jars to the Vietnamese Go Sanh kilns of the fifteenth and sixteenth centuries. Brown (1988: plate 22) likewise suggests that Tradition 2 jars were produced at the Go Sanh kilns, although at the time all three authors were writing the central Vietnamese kilns had not yet been explored archaeologically. Several rim profiles from the kiln sites of central Viet Nam resemble Tradition 2 in rim shape and shoulder angles (Morimoto and Ohashi 2002: fig. 5, no. 16; Yamamoto et al. 1993: 176, figs. 1, 3-4).

In contrast, Tradition 3 vessels appear to be the earliest in the Guthe assemblage. They were recovered from the twelfth-century Jepara shipwreck, off the coast of Java (Djuana and McKinnon 2005 :137, figs. 10-11). Likewise, Valdes et al. (1992:102109, plates 16-19, 26-29) attribute similar forms to the Quanzhou kiln complex in China dating to the twelfth through fourteenth centuries (Song and Yuan dynasties). Tradition 3 vessels are also attributed by Lam et al. to the twelfth-fourteenth-century Quanzhou kilns (1985:110, nos. 229a and b). Simple vertical handles were produced in the kilns of central Viet Nam (Koezuka et al. 1996:24-25, figs. 12, 14); however, similar handles are found throughout Southeast Asia.

Due to sample size and diversity, this is the weakest group identified in my research, both compositionally and technologically. Only two of three fragmented vessels with Tradition 3 dragons were compositionally analyzed, and the other three vessels are whole pots and were not included in the INAA study.

The Kingany vessel (alternative Tradition 1) clusters compositionally with Traditions 2 and 3 in the INAA and the LA-ICP-MS analyses, and was therefore produced at production locale B/C (Sinopoli et al. 2006). Originally excavated from a cavity in the washroom of a "bourgeois" house and presumably used as a receptacle for wash water by the residents, the deposition context of the jar dates to the fifteenth century (Vérin 1972:295-301; Wright et al. 1996). While this date generally matches the late temporal period for Tradition 1 jars presented above, the vessel has other characteristics that indicate production at locale $\mathrm{B} / \mathrm{C}$, notably a rim identical to those from Tradition 2 and large imperfections in the glaze and potentially in the paste, characteristics found in other vessels from locale B/C and not in vessels from locale A. This Kingany vessel shows that the dragon jar external "styles" may not have been uniquely produced in one place, with only subtle differences in the production process differentiating locales.

\section{Production Locale D (Tradition 4)}

Dragon jars from both Traditions 4A and 4B are sourced to the same production locale. However, shipwreck and excavation data indicate that they may be temporally distinct.

Several lines of evidence place Tradition $4 \mathrm{~A}$ in the mid-fifteenth century with a production locus in central Viet Nam (Diem 2004:478, fig. 2; Loviny 1996:50, 
100-101, figs. 7-8). The majority of dragon jars from the Pandanan Island shipwreck noted above belong to Tradition 4A and vessels resembling Tradition 4A jars with similar profiles and necks, red paste, and identical motifs have been recovered from fifteenth-sixteenth-century central Vietnamese kiln sites (Aoyagi 2002: fig. 4, no. 20; Koezuka et al. 1996:24, 27, figs. 4-5, 50; Yamamoto et al. 1993:176, figs. 6, 12, 15-16; $1995: 50,85,100$, figs. 7-8). Recent work by Diem also indicates that the mold-impressed vessels from our Tradition 4A are identical to those found at the kilns and the Pandanan shipwreck (Diem 1999, 2004).

Both Valdes et al. (1992:141) and Harrisson (1986: plates 108-112) date Tradition $4 \mathrm{~B}$ vessels to the seventeenth to eighteenth centuries, although Valdes et al. attribute them to South China and Harrisson to Viet Nam (which is supported by the data on similarly sourced Tradition 4A). Several indirect lines of evidence also suggest that Tradition 4B vessels post-date those of Tradition 4A. First, Traditions 4A and $4 \mathrm{~B}$, compositionally sourced to the same place, do not co-occur in the Pandanan shipwreck, which may suggest temporal variability within the production locale. The mold-applied techniques of Tradition 4B also appear to be most similar to ones used for Tradition 2 jars found in the A.D. 1600 San Diego shipwreck (Desroches et al. 1996), perhaps suggesting that mold-applied techniques may post-date moldimpressed. The shift in techniques could have been a regional diachronic trend that occurred at multiple production centers, but perhaps at slightly different times.

\section{SUMMARY}

Production locale A was likely in southern China, and our jars were produced in the thirteenth to fifteenth centuries. Production locale D was situated in central Viet $\mathrm{Nam}$, and our jars are products of the fifteenth to at least the seventeenth centuries. Evidence for the provenience of production locale $\mathrm{B} / \mathrm{C}$ is more complicated, with its intra-center diversity and longevity of production (see Table 9). Several archaeological dates are available for the diverse traditions practiced at the locale: the twelfth-century Jepara shipwreck date (Tradition 3), the terminus ante quem fifteenth-century date for the deposition in Madagascar of the Kingany vessel (alternative Tradition 1), the mid-

Table 9. Proposed Chronology of Dragon Jar Traditions

\begin{tabular}{|l|c|l|l|}
\hline PRODUCTION TRADITION & PRODUCTION CENTER & \multicolumn{1}{|c|}{ KILN LOCATION } & \multicolumn{1}{c|}{ DATES } \\
\hline 1 & A & China & c. A.D. 1200-before 1450 \\
\hline 3 & B/C & China & c. A.D. 1100-1400 \\
\hline Kingany Vessel & B/C & China & before A.D. 1450 \\
\hline 2 & B/C & China & A.D. 1450-1600 \\
\hline $4 A$ & D & Central Viet Nam & c. A.D. 1450 to (?) \\
\hline 4B & D & Central Viet Nam & c. A.D. 1600-1800 (?) \\
\hline
\end{tabular}


fifteenth-century date for the Pandanan vessels (early Tradition 2), and an A.D. 1600 date for the San Diego's typical Tradition 2 vessels. As discussed above, the Pandanan vessel may mark the transition between techniques at the center, as it exhibits a Tradition 2 primary motif and associated Tradition 1 handle. Since no vessels that match this transitional form are found in the Guthe Collection, it can be inferred that our Tradition 2 jars post-date the mid-fifteenth century. The hand-coiled decorative techniques that characterize the Tradition 3 vessels likely pre-date both, based on the Jepara shipwreck and historical attributions, and their absence from later shipwrecks. Production locale B/C thus comprises a long temporal sequence of dragon jars, starting with Tradition 3 vessels and the Kingany vessel made between the twelfth and fifteenth centuries (none have been found in shipwrecks of the fifteenth and sixteenth centuries), and Tradition 2 vessels from the fifteenth, sixteenth, and likely into the seventeenth century.

With our expanding knowledge of the central Vietnamese kilns and their products, little evidence for primary motifs and plastic handle decoration from locale B/C has been found, despite some similar rim forms. At present, due to the longevity of this production center and similar stylistic ideals (vertical plastic handles, glaze colors), the location of the region responsible for production should be sought in China, and according to several researchers, likely in Guangdong, Southeast China (Lam et al. 1985; Valdes et al. 1992). However, despite similar stylistic variables, technological differences suggest that locales $\mathrm{A}$ and $\mathrm{B} / \mathrm{C}$ were organized very differently and exhibit a large degree of variability in the nature of production, despite their potentially common political origin.

Based on the above information, we can identify a broad trend in dragon jar decoration techniques from coil-based, to mold-impressed, to mold-applied techniques. Early jars (Traditions 1 and 3) have dragons on the shoulder that are later expanded onto the body in Traditions 2 and 4, and vessel thickness increases with mold-applied techniques. Concurrently, the visual appearance of the dragon and other motifs also changed, with potters employing more elaborate and larger decorations over time. Evidence from the Pandanan shipwreck also suggests that mold-applied techniques in production locale $\mathrm{B} / \mathrm{C}$ (Tradition 2) likely pre-date a similar technological development (4A to $4 \mathrm{~B}$ ) at production locale D. Glaze composition did not change over time within individual production locales.

The evidence from the INAA and LA-ICP-MS compositional analyses for production locales $\mathrm{A}$ and $\mathrm{B} / \mathrm{C}$ suggests that different production centers may have occasionally or always produced visually similar, but not technologically exact, dragon and handle decoration motifs, suggesting the need to record all technological variables available on a sample. The results of this study establish that non-visual categories, such as the clay, inclusions, glaze recipe, and paste treatment, as well as technological variations in seemingly identical primary motifs and handles are useful indicators to separate production locales, due to differential organization of production.

\section{TESTING THE PROPOSED CHRONOLOGY AGAINST THE GUTHE COLLECTION}

The sequence presented above was tested against the Guthe Collection both spatially and by site type (see Table 10): 
Table io. Distribution of Dragon Jars From the Guthe Collection

\begin{tabular}{|c|c|c|c|c|c|c|}
\hline \multirow{2}{*}{$\begin{array}{l}\text { PRODUCTION } \\
\text { TRADITION }\end{array}$} & \multicolumn{2}{|r|}{ CAVE SITES } & \multicolumn{2}{|c|}{ BURIAL GROUNDS } & \multicolumn{2}{|c|}{ MISCELLANEOUS } \\
\hline & $\mathrm{N}$ & ISLANDS & $\mathrm{N}$ & ISLANDS & $\mathrm{N}$ & ISLANDS \\
\hline 1 & 4 & $\begin{array}{l}\text { North Mindanao, } \\
\text { West Cebu, South } \\
\text { Bohol }\end{array}$ & 3 & Bohol, Cebu & 4 & $\begin{array}{l}\text { Camaguin, } \\
\text { Siquijor, Jolo }\end{array}$ \\
\hline 2 & 14 & $\begin{array}{l}\text { Oacan, Samar, South } \\
\text { Cebu, North Masbate, } \\
\text { Calamianes }\end{array}$ & 15 & $\begin{array}{l}\text { Koulan, } \\
\text { Zamboanga, } \\
\text { Cebu, South } \\
\text { Cebu }\end{array}$ & 6 & $\begin{array}{l}\text { Siquijor, } \\
\text { Masbate, South } \\
\text { Cebu, North } \\
\text { Masbate }\end{array}$ \\
\hline 3 & 1 & Masbate & 3 & $\begin{array}{l}\text { North Masbate, } \\
\text { Cebu, Negros }\end{array}$ & 2 & $\begin{array}{l}\text { West Cebu, } \\
\text { Jolo }\end{array}$ \\
\hline $4 \mathrm{~A}$ & 2 & Suluan, Zamboanga & 5 & Koulan & None & None \\
\hline $4 B$ & None & None & 3 & Bohol, Koulan & None & None \\
\hline
\end{tabular}

Tradition 1 vessels are found in cave sites, isolated graves, open-air burial grounds, and miscellaneous sites in the Philippines. All jars except for that from Jolo are located around the southern interior seas of Visayan, Camotes, and Mindanao (see Figure 5).

Tradition 3 jars were recovered from a cave, open-air burial grounds, an isolated grave, and a miscellaneous context. All except for the vessel from Jolo are located around the southern interior seas of Visayan, Camotes, and Mindanao (see Figure $6)$.

Tradition 2 dragon jars are found in a larger diversity of locations throughout the central and southern islands, including caves, open-air burial sites, a single grave site, and some in miscellaneous contexts. Jars were recovered from sites located throughout the southern archipelago, with most (74\%) deposited away from the central interior seas of Visayan, Camotes, and Mindanao (see Figure 7).

Tradition 4 vessels can be divided between $4 \mathrm{~A}$ and $4 \mathrm{~B}$ jars, with the $4 \mathrm{~A}$ vessels deposited in both cave and open-air burial sites, and $4 \mathrm{~B}$ jars in only open-air burial sites. Tradition 4 vessels in general seem to mirror the spatial pattern found for Tradition 2 , with only one out of ten found on islands around the central interior seas of Visayan, Camotes, and Mindanao (see Figure 8).

The co-occurrence of various dragon jar production traditions confirms the temporal patterns suggested above. Traditions 1 and 3 co-occur on Jolo (M28) as well as at the one burial site that they have been attributed to on Cebu (B49). Vessels from these groups were not found in sites that yielded Tradition 2 and 4 jars. The spatial extent of their collective distribution is around the central interior seas of the southern archipelago.

Tradition 2 and 4 vessels co-occur at a cave site (C23 on Suluan) and one burial site on Koulan (B4). A Tradition 2 vessel and two Tradition 4A vessels were found in the cave, mirroring the co-occurrence of similar vessels on the Pandanan shipwreck. 


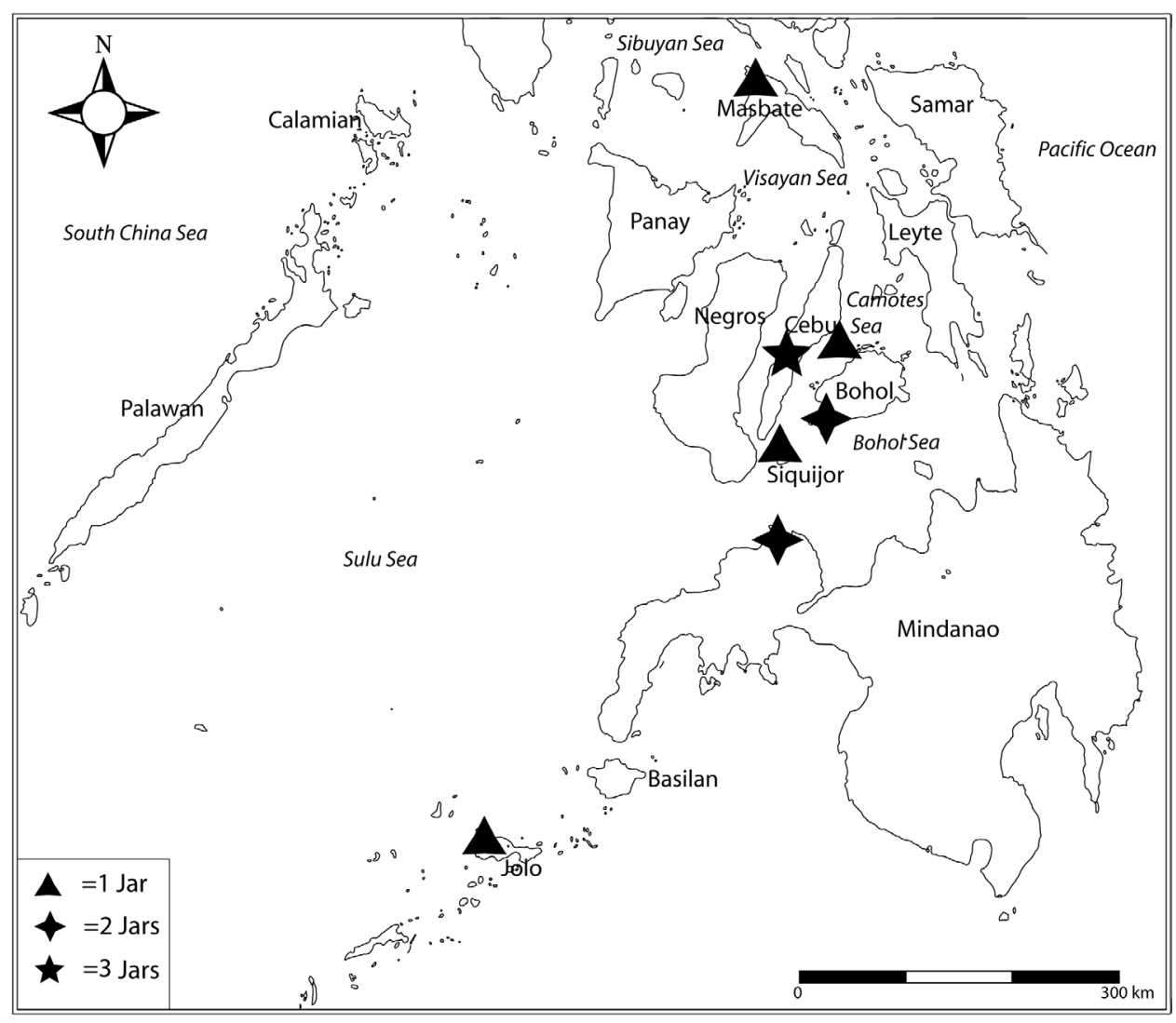

Fig. 5. The distribution of Tradition 1 dragon jars.

The large burial site of B4 contains ten vessels from Tradition 2, five vessels from Tradition 4A, and two vessels from Tradition 4B. It would seem from this additional cooccurrence that Tradition 2 and 4A vessels were likely produced at least starting in the mid-fifteenth century with Tradition 2 vessels in production until at least 1600 as exhibited on the San Diego. It is likely that Tradition 4B, which does not occur solely with Tradition 2 without Tradition 4A also present, does post-date Tradition 4A, with the production center starting to use mold-applied techniques at the earliest following the mid-fifteenth century.

Jars from Traditions 2 and 4 were found in a wider variety/different places in the southern Philippine archipelago than Traditions 1 and 3, with two open-air cemeteries with many dragon jars appearing on Koulan and Samar, away from the central interior sea area where Tradition 1 and 3 vessels were almost exclusively found. If the temporal sequence of jar types is valid, the patterns suggest a spatial expansion in jar distribution and, perhaps, large burial sites. In short, an expansion in the trade is seen in the wider diversity of islands with access to dragon jars over time, including those away from the central seas (see Figures 9 and 10). This pattern is further enforced by evidence of porcelain distribution described by $\mathrm{Li}$ in this volume. 


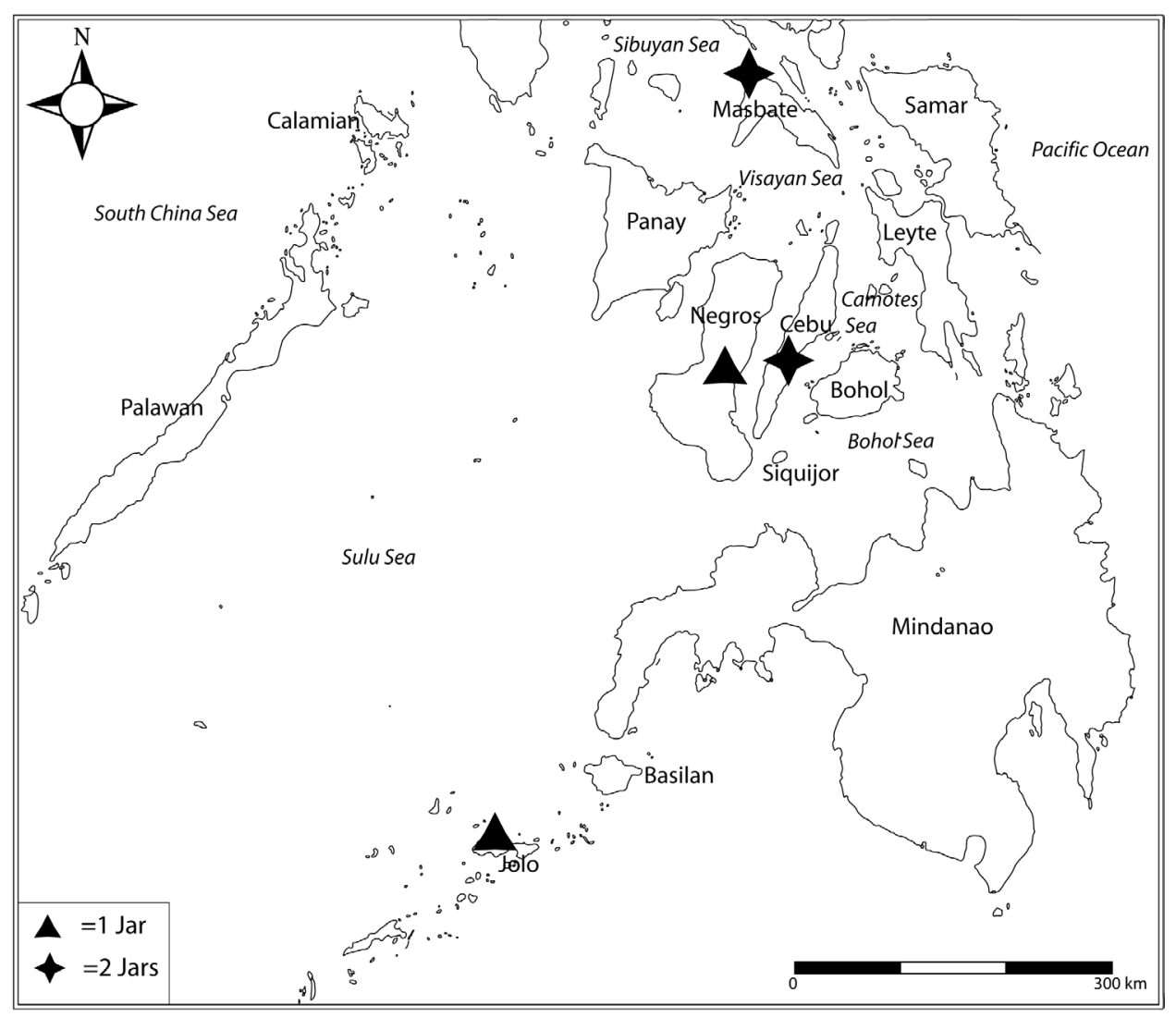

Fig. 6. The distribution of Tradition 3 dragon jars.

\section{DISCUSSION}

If the postulated Chinese origin of Tradition 1 is considered, then these jars may be indicative of Late Song, Yuan, and Early Ming involvement in the Philippines. Tradition 4 jars, with a well-substantiated provenience to Champa territories of Central Viet Nam may represent the general diversification of the interregional trade during the mid- to late fifteenth century, as well as evidence of the "Ming Gap" postulated by Roxanna Brown (2004). The situation is more complicated for products of production locale $\mathrm{B} / \mathrm{C}$, as vessels seem to span the entire temporal sequence from Song and Yuan, to Ming commerce, and likewise the entire technological span from the hand-coiled decorations of Tradition 3 to mold-impressed in the style of Tradition 1 (the Kingany vessel), and lastly mold-applied Tradition 2 vessels. The longevity of production suggests a Chinese origin for this group, as some vessels were clearly produced during the Song and Yuan periods, and the relatively large number of Tradition 2 vessels may mark the re-emergence of foreign trade during the Late Ming dynasty (see Brown 2004). Interestingly, the absence in our collection of the transitional Tradition 2 vessels that were found in the Pandanan wreck may provide additional evidence for the Ming Gap of Chinese-produced jars in the Philippines, as 


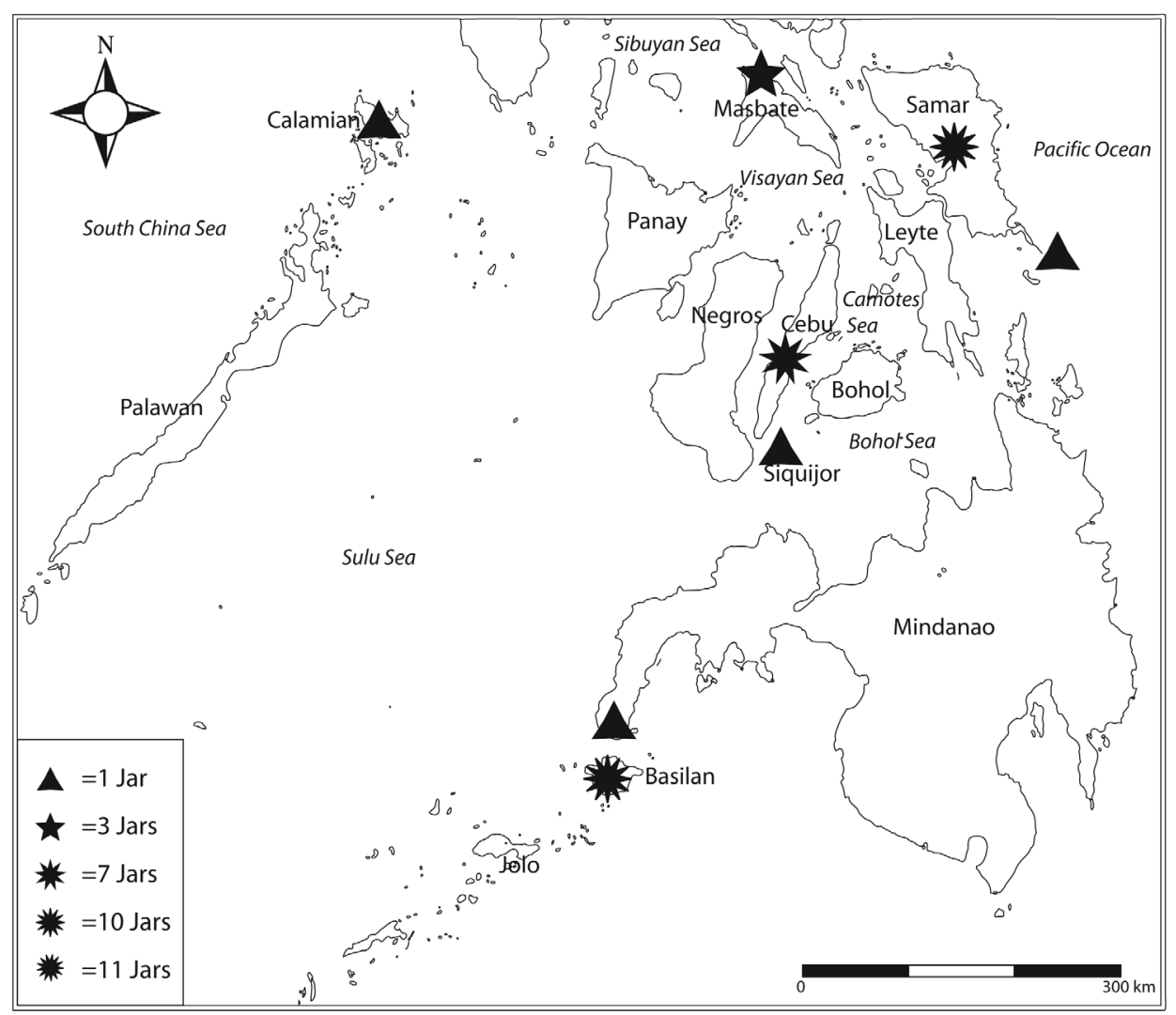

Fig. 7. The distribution of Tradition 2 dragon jars.

Guthe recovered Vietnamese Tradition 4A, but not the contemporaneous Chinese jar. Understanding the exact provenience of locales $\mathrm{A}$ and $\mathrm{B} / \mathrm{C}$ will reveal a great deal about the organization of trade during this period. For example, perhaps two production centers in southern China were responsible for Tradition 1 vessels, with only one developing (or continuing) Tradition 2 techniques.

Co-occurrence of only contemporary jar production traditions within the twopart temporal sequence at sites around the Philippines suggests that the curation of jars as heirlooms may not have been significant historically, with individuals aiming to obtain their own jars and be buried with them. However, without a detailed understanding of the regional distribution and nature of the various societies in the Philippines during this period, it is possible that there were diverse practices with some groups or individuals gaining prestige through the inclusion of jars in burials and other societies for whom transmission of jars as heirlooms was of social significance.

Several trends in jar technology and decoration may suggest that the secondary life of jars in local sociocultural settings after their use in trade may have influenced their formal and decorative characteristics. For example, jars became thicker over time, and it is possible that these jars were sturdier, and consequently had longer use-lives. 


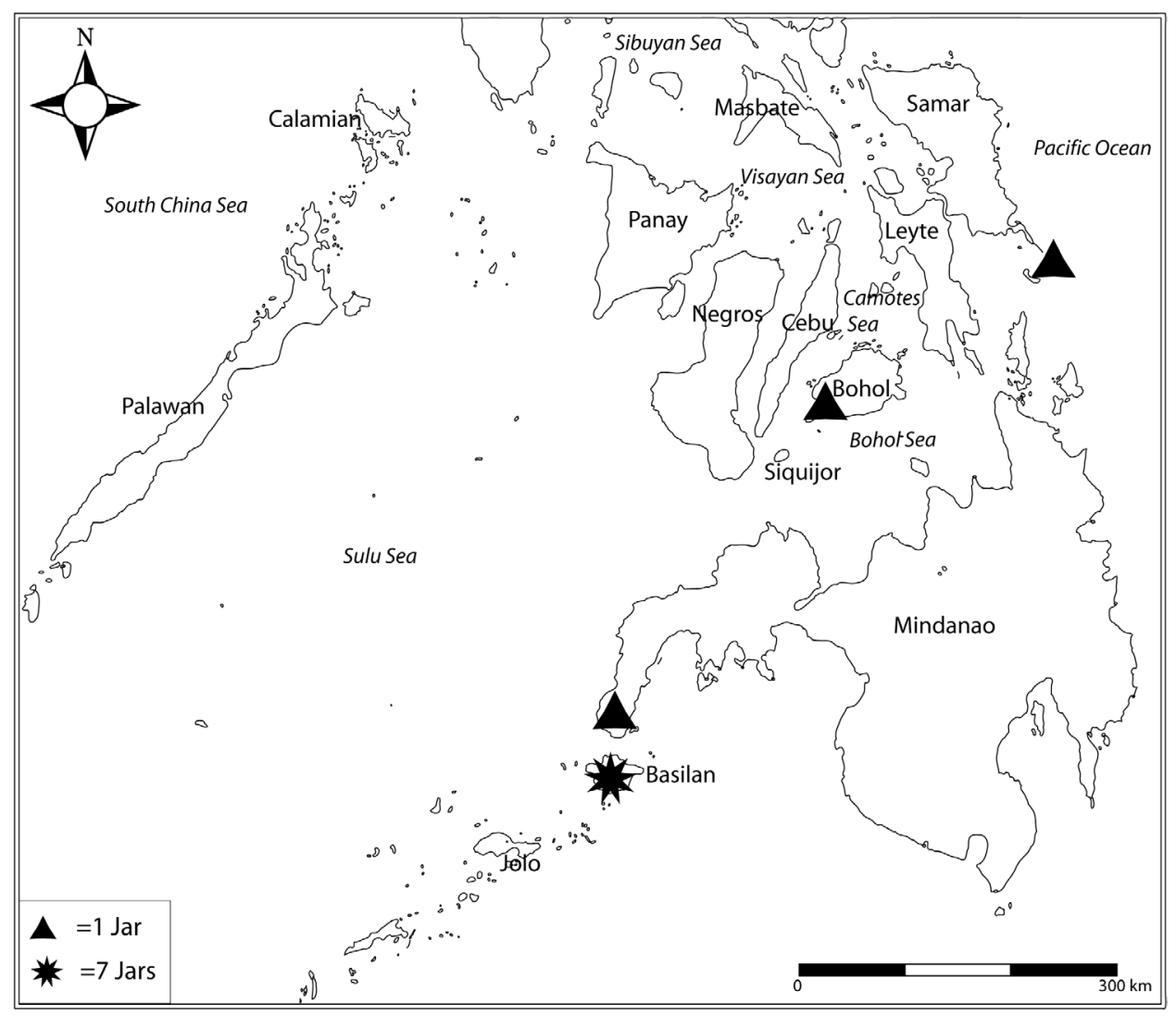

Fig. 8. The distribution of Tradition 4 dragon jars.

Secondly, jars became more elaborately decorated over time, with Tradition 1 and 3 vessels decorated (dragon and occasional incision) on the vessel shoulder, whereas in Traditions 2 and 4 dragons and floral motifs are found from the shoulder all the way to near the vessel base. It is possible that the increase in decoration of storage vessels was influenced by their use as a trade item with added value. In social settings where jars symbolized status, and were themselves ranked historically in some parts of Southeast Asia, it would be unsurprising if producers responded to the desires of locals for more elaborate vessels.

\section{CONCLUSION}

In this article, I have examined a small subset of jars from the Guthe Collection. Nonetheless, its implications are many both for this collection and for larger understandings of second millennium Asian trade and political economies, and the scholarly potential of this little studied vessel category. Through an examination of the techniques responsible for jar production, particularly of decorative elements, multiple production locales have been discerned. Chronologically, the four traditions identified bridge the period from initial indirect Chinese trade in the Philippines during 


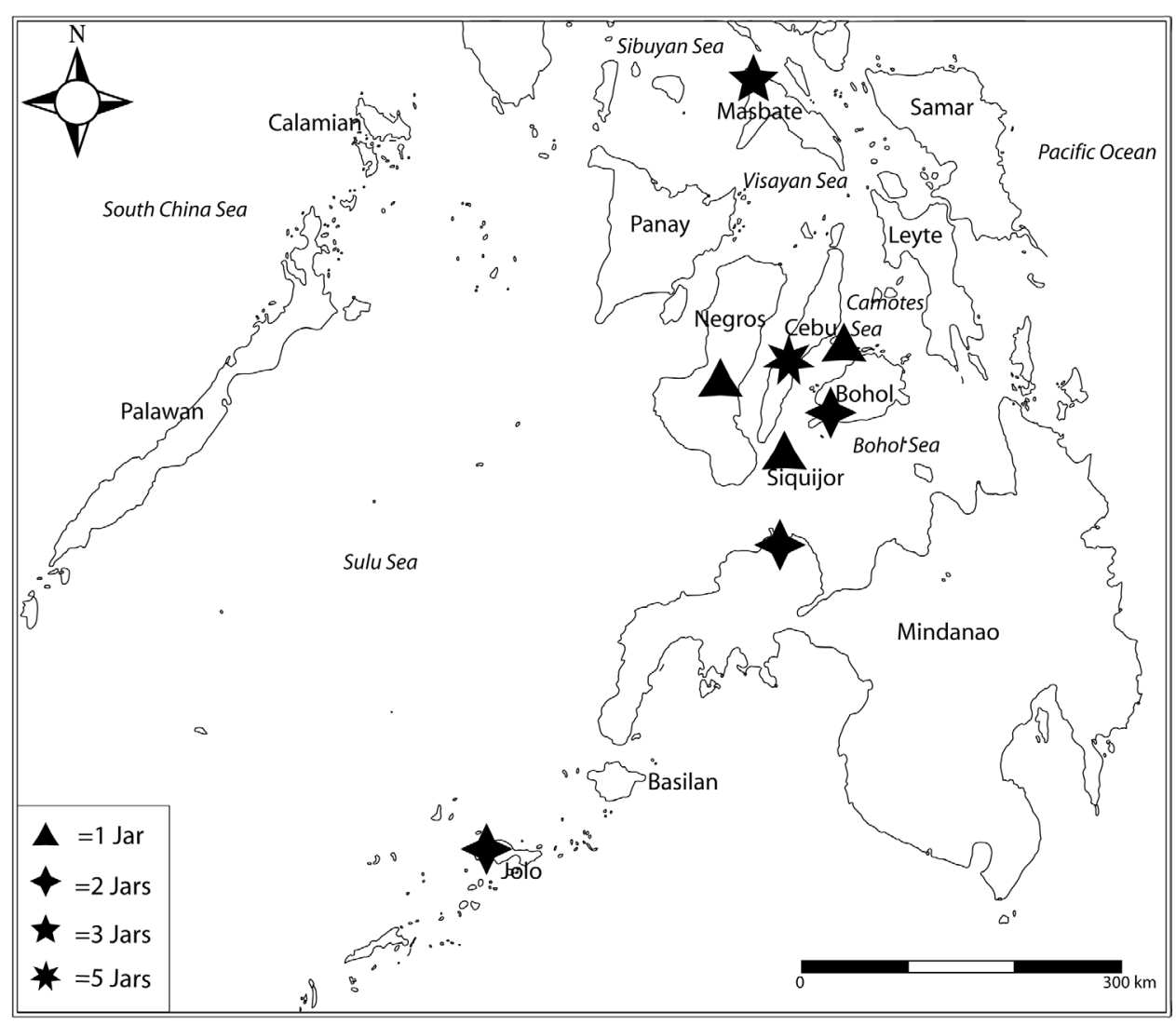

Fig. 9. The distribution of Late Song, Yuan, and Early Ming dragon jars.

the twelfth and thirteenth centuries (Traditions 1 and 3), and the direct and more intense participation (Traditions 1 and 3) of the fourteenth century, to the growing diversification of trading and producing partners beginning after A.D. 1426 (Tradition 4A), and the Spanish conquest of the late sixteenth and early seventeenth centuries (Tradition 2 and likely 4B). These international shifts can be linked to correlated shifts in mortuary programs within the Philippines.

Evidence for differences between production regions may itself be significant, as the organization of production of a single class of vessels may have been different due to the particular political or economic contexts and traditions within which ceramic production was embedded. For example, the intra-group diversity of locales B/C and $\mathrm{D}$ wares suggests that either multiple production groups were in operation simultaneously, or choices changed frequently in various techniques. I would argue that given the wide diversity found even within the dragon motifs themselves, that many different potters were regionally involved in production. As has been discussed throughout the article, the wares from locale D were likely produced in the Champa setting, politically a loose confederation of states, such that jar production may have been noncentralized, or dispersed like the organization of the polity. Consequently, it is possible that a similar organization characterized the area of locale $\mathrm{B} / \mathrm{C}$, and that the 


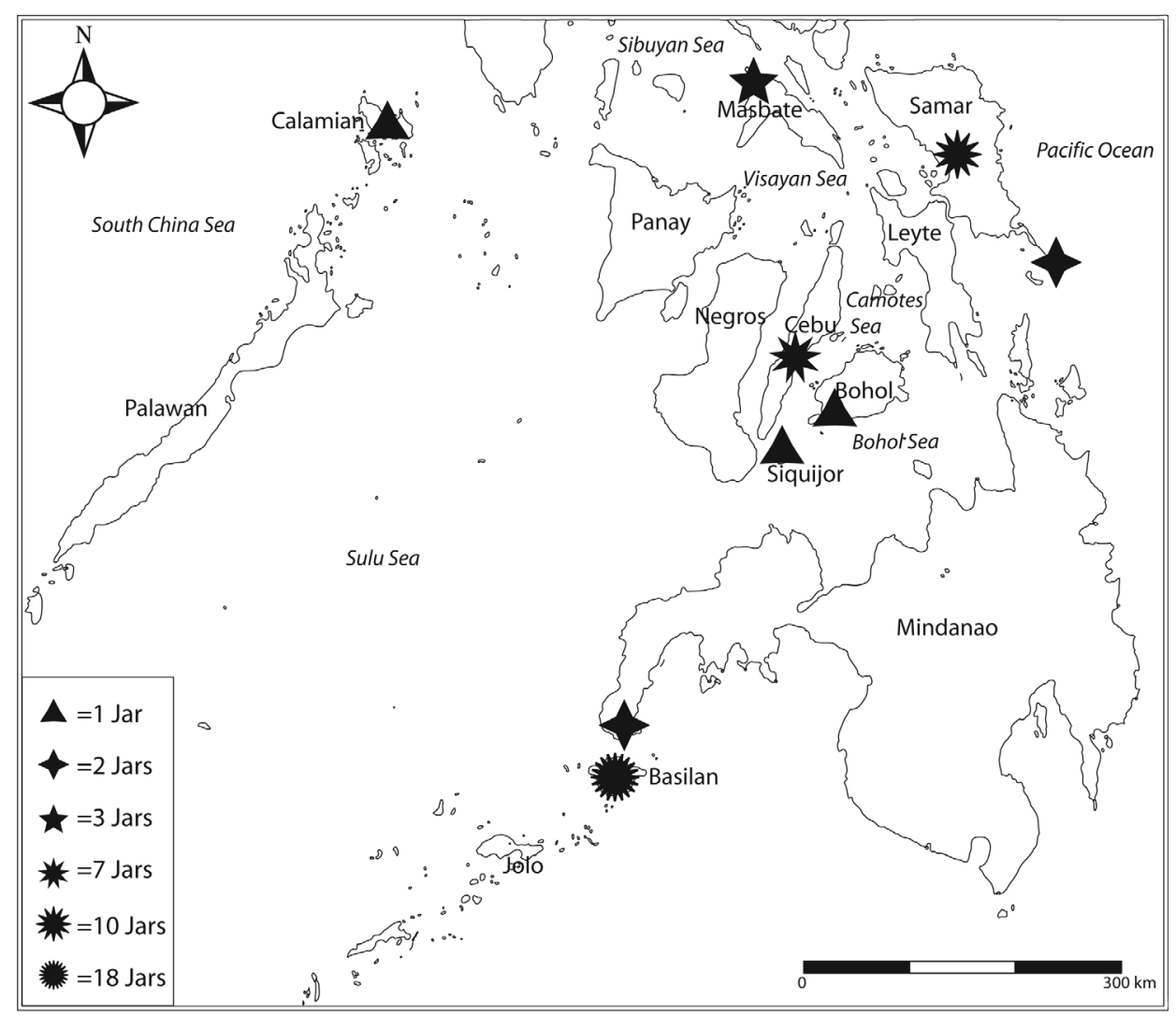

Fig. 10. The distribution of Mid / Late Ming dragon jars.

noncentralized local organization was unmodified by the Chinese state over time. The local political setting at locale A may have been more sociopolitically centralized, as its products were much more homogenous and standardized in paste and decoration. The difference in production between these two proposed areas of China may indicate that the Chinese state practiced some degree of indirect rule within certain economic spheres, with local organizational forms left in place.

While the organization of production may have been left to local decisions, data from the Guthe Collection may support the historically known reorganization of commerce by licensed Chinese merchants with the installation of the Ming Dynasty in A.D. 1368. For example, prior to the Ming Gap there are two production locales active in China, locales $\mathrm{A}$ and $\mathrm{B} / \mathrm{C}$. However, only locale $\mathrm{B} / \mathrm{C}$ carries through to the Middle and Late Ming periods. This may indicate a shift in (and/or reduction in the number of) ports utilized as the state centralized control over trade, and possibly over these cities. However, the consistency in jar manufacture spanning this shift suggests little interest in altering the mode of dragon jar production.

My goal in undertaking the study presented here was to describe the patterns in a small assemblage of dragon jars in hopes of building a chronology of general use. 
Several specific trends in jar production have been identified. These include a general technological development from mold-impressed and hand-coil decorated motifs to mold-applied techniques starting in the mid-fifteenth century. This chronology provides a preliminary point from which diachronic trends in the region can be ascertained. Jars are particularly important to sequence, as storage containers are necessarily associated with all trade events, whereas the products that they carried may have changed frequently. The test described on patterning of dragon jars across time and space and burial type in the Guthe Collection is only a first example of the potential for similar kinds of studies to address research questions in the region.

\section{ACKNOWLEDGMENTS}

Primarily, I would like to thank Carla Sinopoli, who introduced me to dragon jars and has encouraged me throughout this research. Helpful suggestions on early drafts were provided by Henry $\mathrm{T}$. Wright, and the final version was significantly improved by the valuable comments of three anonymous reviewers. This article builds on the foundation of research on the Guthe Collection dragon jars by Robert Brubaker, Cristophe Descantes, Michael D. Glascock, Will Griffin, Hector Neff, Rasmi Shoocongdej, and Robert J. Speakman. Lastly, I particularly wish to acknowledge Louise Cort for providing useful references on recent archaeological research in Viet Nam. Revisions were completed during an ACLS New Faculty Fellows Award, through the support of The Andrew W. Mellon Foundation.

\section{NOTES}

1. Preliminary patterns of this study and a detailed description of the chemical analyses were presented in Sinopoli et al. 2006.

2. Essential information on dragon jar production comes from interviews conducted by Barbara Harrisson with Lau Hua Kee, a potter who produced stoneware jars in Sarawak, Borneo, in the mid 1980's (Harrisson 1986).

\section{REFERENCES CITED}

Aoyagi, Yoji

2002 Excavation of the Go Sanh Kiln Complex: Champa ceramics in the history of maritime route of the Silk Road. In Champa Ceramics: Production and Trade: 5-18, ed. Yogi Aoyagi and Gakuji Hasebe. Tokyo: Study Group of Go Sanh Kiln Sites in Central Vietnam.

Bacus, Elisabeth

1995 Political Economy and Interaction: Late Prehistoric Polities in the Central Philippine Islands. Ph.D. diss. University of Michigan.

Barbosa, Artemio C.

1992 Heirloom jars in Philippine rituals. In A Thousand Years of Stoneware Jars in the Philippines, ed. Cynthia O. Valdes, Kerry Nguyen Long, and Artemio C. Barbosa. Manila: Jar Collectors (Philippines).

BROWN, ROXANNA

1988 The Ceramics of South-East Asia: Their Dating and Identification, 2nd ed. Oxford: Oxford University Press.

2004 The Ming Gap and Shipwreck Ceramics in Southeast Asia. Ph.D. diss. University of California, Los Angeles.

Desroches, Jean-Paul, Gabriel Casal, and Franck Goddio, eds.

1996 Treasures of the San Diego. Manila: National Museum of the Philippines. 
Diem, Allison

1999 Ceramics from Vijaya, Central Vietnam: Internal motivations and external influences (14thlate 15 th century). Oriental Art 45(3):55-64.

2004 Ceramic evidence of ancient maritime relationships between central Viet Nam and the Philippine archipelago. In Southeast Asian Archaeology: Wilhelm G. Solheim II Festschrift: 463-490, ed. Victor Paz. Diliman, Quezon City: The University of the Philippines Press.

Djuana, Atma, and Edmund McKinnon

2005 The Jepara wreck. In Proceedings of the International Conference: Chinese Export Ceramics and Maritime Trade, 12th-15th Centuries: 126-142, ed. Pei-kai Cheng, Guo Li, and Chui Ki Wan. Hong Kong: Chinese Civilization Centre, City University of Hong Kong.

Fox, ROBERT

1959 The Calatagan excavations: Two fifteenth century burial sites in Batangas, Philippines. Philippine Studies 7(3):325-390.

1967 The archaeological record of Chinese influences in the Philippines. Philippine Studies 15(1): 41-62.

Grave, Peter, and Michael Maccheroni

2009 Characterizing Asian stoneware jar production at the transition to the Early Modern period, 1550-1650. In Scientific Research on Historic Asian Ceramics: Proceedings of the Fourth Forbes Symposium at the Freer Gallery of Art: 186-206, ed. Blythe McCarthey, et al. London: Archetype Publications.

GUY, JOHN

1986 Oriental Trade Ceramics in South-East Asia: Their Dating and Identification. Oxford: Oxford University Press.

Harrisson, Barbara

1986 Pusaka: Heirloom Jars of Borneo. Singapore: Oxford University Press.

Hegmon, Michelle

1992 Archaeological research on style. Annual Review of Anthropology $21: 517-536$.

JUNKer, LAura LeE

1990 Long-Distance Trade and the Development of Socio-Political Complexity in Philippine Chiefdoms of the First Millennium to Mid-Second Millennium A.D. Ph.D. diss. The University of Michigan, Ann Arbor.

1999 Raiding, Trading, and Feasting: The Political Economy of Philippine Chiefdoms. Honolulu: University of Hawai'i Press.

Koezuka, Takayasu, Shuji Ninomiya, Mamoru Aboshi, and Kazuo Tamasaki

1996 Scientific study on the ancient Vietnamese ceramics: Study mainly based on the shards collected and excavated at Go Sanh Kilns in central Vietnam. Journal of East-West Maritime Relations $4: 1-33$.

Lam, Peter Y. K., John Carswell, Tristan Russell, Mohammed Bin Abu Bakar Mokhtar, K. K. Kwan, AND JeAn Martin

1985 A Ceramic Legacy of Asia's Maritime Trade: Song Dynasty Guangdong Wares and Other 11th to 19th Century Trade Ceramics Found on Tioman Island, Malaysia. Oxford: Oxford University Press.

LEMONNIER, PierRe

1992 Elements for an Anthropology of Technology. Anthropological Papers 88. Ann Arbor: University of Michigan Museum of Anthropology.

Leroi-Gourhan, ANDrÉ

1943 Évolution et Technique, vol. 1: L’Homme et la Matière. Paris: Albin Michel.

1945 Évolution et Techniques, vol. 2: Milieu et Techniques. Paris: Albin Michel.

Li, MiN

n.d. Fragments of Globalization: Chinese Blue-and-White Porcelain in the Early Colonial Philippines. Prelim. paper available from the Asia Range, Museum of Anthropology, University of Michigan.

LONG, KerRy NGUYeN

1992 History behind the jar. In A Thousand Years of Stoneware Jars in the Philippines, ed. Cynthia O. Valdes, Kerry Nguyen Long, and Artemio C. Barbosa. Manila: Jar Collectors (Philippines). 
Loviny, Cristophe

1996 The Pearl Road: Tales of Treasure Ships. Makati City, Philippines: Asiatype and Cristophe Loviny.

Mauss, MARCEL

1954 The Gift: Forms and Functions of Exchange in Archaic Societies, trans. Ian Cunnison. London: Cohen and West.

Morimoto, Asako, and Koji Ohashi

2002 An excavation and investigation of Go Sanh Kilns No. 2 and 3 in Binh Dinh Province, Vietnam. In Champa Ceramics: Production and Trade: 37-59, ed. Yoji Aoyagi and Gakuji Hasebe. Tokyo: Study Group of Go Sanh Kiln Sites in Central Vietnam.

Quimpo, EMiLie

1982 Diving for a shipload of porcelain. Filipinas Journal of Science and Culture 4:33-48.

ReID, ANTHONY

1993 Southeast Asia in the Age of Commerce 1450-1680, vol. 2: Expansion and Crisis. New Haven: Yale University Press.

Sinopoli, Carla M., Stephen Dueppen, Robert Brubaker, Cristophe Descantes, Michael D.

Glascock, Will Griffin, Hector Neff, Rasmi Shoocongdej, and Robert J. Speakman

2006 Characterizing the stoneware "dragon jars" in the Guthe Collection: Chemical, decorative, and formal patterning. Asian Perspectives 45(2):243-282.

SOLHEIM, WILHELM

2002 The Archaeology of the Central Philippines: A Study Chiefly of the Iron Age and its Relationships, 2nd ed. Manila: University of the Philippines.

Stark, Miriam T., ED.

1998 The Archaeology of Social Boundaries. Washington D.C.: Smithsonian Institution Press.

Valdes, Cynthia O.

1993 Martaban jars found in the Philippines. Arts of Asia 22:63-74.

Valdes, Cynthia O., and Larry A. Alba

1993 Jars on board. In Saga of the San Diego, ed. Cynthia O. Valdes. Manila: Concerned Citizens for the National Museum Press.

Valdes, Cynthia O., Kerry Nguyen Long, and Artemio C. Barbosa

1992 A Thousand Years of Stoneware Jars in the Philippines. Manila: Jar Collectors, Philippines, and the National Museum of the Philippines.

VÉRIN, PIERRE

1972 Les Echelles Anciennes Du Commerce Sur Les Cotes Nord De Madagascar. Paris: L'Université de Paris I.

VICKERY, MiCHAEL

2009 A short history of Champa. In Champa and the Archaeology of My Son (Vietnam): 45-60, ed. Andrew Hardy, Mauro Cucarzi, and Patrizia Zolese. Singapore: National University of Singapore.

WIESSNER, POLLY

1983 Style and social information in Kalahari San projectile points. American Antiquity 49:253-276.

1984 Reconsidering the behavioral basis for style: A case study among the Kalahari San. Journal of Anthropological Archaeology 3 : 190-234.

1985 Style and isochrestic variation? A reply to Sackett. American Antiquity 50:160-166.

WOBST, H. MARTIN

1977 Stylistic behavior and information exchange. In For the Director: Research Essays in Honor of James B. Griffin: 317-342, ed. Charles E. Cleland. Anthropological Papers 61. Ann Arbor: University of Michigan Museum of Anthropology.

Wright, Henry T., Pierre Vérin, Ramilisonina, David Burney, Lida P. Burney, and Katsumi Matsumoto

1996 The evolution of settlement systems in the Bay of Boeny and the Mahavavy River Valley, northwestern Madagascar. Azania $31: 37-73$.

Yamamoto, Nobuo, Gakuji Hasebe, Yoji Aoyagi, and Hidefumi Ogawa

1993 The chronological study of Vietnamese wares and the excavation of the old kiln site in Champa. Journal of Sophia Asian Studies 11 : 163-180. 


\begin{abstract}
This article presents the results of a detailed analysis of four dragon jar groups found in the Guthe Collection at the University of Michigan Museum of Anthropology. Dragon jars are a class of decorated stoneware storage vessels that were employed in trade throughout Southeast Asia during the second millennium A.D. The jars in this study, recovered from mortuary contexts, are a unique data set due to their wide deposition throughout the southern Philippines. An exploration of intra-group and inter-group patterning has revealed temporal patterns and likely production locales for dragon jar manufacture over the course of the twelfth to seventeenth centuries. These temporal and spatial trends are then applied to the Guthe Collection to examine jar distribution throughout the Philippines over the critical period spanning the emergence of largescale international commerce in the region. This study contributes a well-defined chronology for a commonly found material class, as well as knowledge of regional trading patterns. KeYwords: Southeast Asia, ceramic classification, dragon jars, trade.
\end{abstract}

